\title{
Predicting Issuer Credit Ratings Using a Semiparametric Method ${ }^{*}$
}

\author{
Ruey-Ching Hwang ${ }^{\mathrm{a},}{ }^{\dagger}$, Huimin Chung ${ }^{\mathrm{b}}$, and C.K. Chu ${ }^{\mathrm{c}}$ \\ ${ }^{\mathrm{a}}$ Department of Finance, National Dong Hwa University, Hualien, Taiwan \\ ${ }^{\mathrm{b}}$ Graduate Institute of Finance, National Chiao Tung University, Hsinchu, Taiwan \\ ${ }^{\mathrm{c}}$ Department of Applied Mathematics, National Dong Hwa University, Hualien, Taiwan
}

\begin{abstract}
This paper proposes a prediction method based on an ordered semiparametric probit model for credit risk forecast. The proposed prediction model is constructed by replacing the linear regression function in the usual ordered probit model with a semiparametric function, thus it allows for more flexible choice of regression function. The unknown parameters in the proposed prediction model are estimated by maximizing a local (weighted) log-likelihood function, and the resulting estimators are analyzed through their asymptotic biases and variances. A real data example for predicting issuer credit ratings is used to illustrate the proposed prediction method. The empirical result confirms that the new model compares favorably with the usual ordered probit model.
\end{abstract}

JEL classification: C14; G20

Keywords: Industry effect; Issuer credit rating; Market-driven variable; Ordered linear probit model; Ordered semiparametric probit model

\footnotetext{
* The research was supported by National Science Council, Taiwan, Republic of China.

†Corresponding author. Email: rchwang@mail.ndhu.edu.tw; Tel: +886-38633139; Fax: +886-38633510.
} 


\section{Introduction}

Credit ratings play an important role in capital markets. Under the New Basel Capital Accord (Basel II), credit ratings will play an even more central role than they have so far. There are two basic types of credit ratings, the bond rating and the issuer credit rating. While the former measures the likelihood of the default or delayed payment of a bond issue, the latter is an overall assessment of the creditworthiness of a company. Currently, there are many widely recognized credit rating agencies, such as Moody's Investors Service and Standard and Poor's Ratings Services (S\&P’s), etc. They routinely provide credit ratings for bonds and companies.

This study focuses on the S\&P's long-term issuer credit rating (LTR). According to the definition given by S\&P's, the LTR focuses on the obligor's capacity and willingness to meet its long-term financial commitments. Based on the Compustat North America (COMPUSTAT) database, in year 2007, there were 8010 companies listed on the New York Stock Exchange, American Stock Exchange, or NASDAQ. However, among those 8010 companies, there were only $18.96 \%$ (1519) companies having S\&P's LTRs. This result indicates that most of companies listed on those stock exchanges do not have S\&P's LTRs, which makes their rating predictions quite valuable to practitioners and regulators. Accordingly, the purpose of this paper is to forecast ratings for those companies “without” S\&P's LTRs. For two reasons, we do not pursue the issue of rating forecast for companies “with” S\&P’s LTRs. First, if a company is once rated by S\&P’s, then it will be rated again unless a special event, for example bankruptcy, happens to the company. Second, the continuously rated companies have relatively unchanged rating categories in general (Galil, 2003; Pettit et al., 2004). Thus it seems less interesting in predicting the ratings of these companies.

There are several well-known statistical techniques for constructing credit rating predictions. These techniques include multiple regression analysis (Horrigan, 1966; Pouge and Soldofsky, 1969; West, 1970), multiple discriminant analysis (Pinches and Mingo, 1973, 1975; Altman and Katz, 1976), ordered linear probit model (OLPM; Kaplan and Urwitz, 1979; Ederington, 1985; Gentry et al., 1988; Hwang et al., 2008), and ordered and unordered linear logit models (Ederington, 1985), etc. Altman et al. (1981) provides a detailed introduction of statistical classification models. The common principal of these approaches is that they are developed using single-period data. Credit rating forecasting models based on multiple-period data with independent assumption include, for example, Blume et al. (1998), Poon (2003), and Güttler and Wahrenburg (2007) employing the idea of OLPM. Other approaches based on machine learning techniques, for example, Bayesian networks (Wijayatunga et al., 2006) and support vector machines and neural networks (Huang et al., 2004) were also considered in the literature for credit rating prediction. 
To forecast S\&P's LTRs, the prediction methods based on OLPM and an extension of OLPM will be used in this paper. ${ }^{1}$ The OLPM is simply constructed by imposing a linear regression relationship between S\&P's LTRs and predictor variables. Its important parameters are determined by maximizing a log-likelihood function. However, if the underlying regression function is not linear, then the advantages of OLPM in explaining and predicting will not be realized. To avoid this potential pitfall, we show in this paper that the idea of semiparametric logit model in Hwang et al. (2007) can be directly extended to OLPM. Specifically, we shall propose an ordered semiparametric probit model (OSPM) for credit rating prediction by replacing the linear regression function in OLPM with a semiparametric function. The proposed OSPM is built on the works of OLPM but needs not assuming any parametric form for the regression function. Thus it is much more flexible in modeling the regression function. Furthermore, the proposed method is developed under the concept of local likelihood, and it turns out that the important parameters in OSPM can be estimated by maximizing a local (weighted) log-likelihood function. Thus the required computation for OSPM is as simple as that for OLPM.

To apply OLPM and OSPM to predict S\&P's LTRs, the twenty-four potential predictors in Hwang et al. (2008) for studying important predictors of S\&P’s LTRs in year 2005 were considered in our data analysis section. These variables include four market-driven variables (Shumway, 2001; Bharath and Shumway, 2008), nineteen accounting variables (Altman, 1968; Poon, 2003; Pettit et al., 2004), and industry effects (Chava and Jarrow, 2004; Pettit et al., 2004). The studied data were collected from COMPUSTAT and Center for Research in Security Prices (CRSP) databases. Our sample consisted of 779 companies receiving S\&P’s LTRs in April 2007 and having complete values of the twenty-four potential predictors. The sample was further divided into the estimation sample and holdout sample based on the longevity of S\&P's LTR (Hwang et al., 2008). ${ }^{2}$ According to S\&P’s Research Insight North America Data Guide (2004, p. 54), S\&P's began to use the term LTR on September 1, 1998. Companies receiving S\&P’s LTRs

\footnotetext{
${ }^{1}$ Due to the superiority in explaining and predicting, OLPM has been adopted for multiple-class prediction by a number of studies such as Kaplan and Urwitz (1979) and Gentry et al. (1988), etc. Also, the test procedure of sample selection bias is only available for OLPM (Greene, 2002). On the other hand, it is not suggested using discrete explanatory variables in multiple discriminant analysis (Johnson and Wichern, 2002, p. 641). In this paper, industry effects on S\&P’s LTR were estimated through coefficients of six industry indicator variables. Given these industry indicator variables, it is not adequate to use multiple discriminant analysis to predict S\&P’s LTRs.

${ }^{2}$ Given the pool of companies with S\&P's LTRs, our estimation companies solely correspond to the rated ones, and our holdout companies the newcomers. Their purified composition agrees with our purpose to forecast ratings for companies without S\&P's LTRs. On the other hand, one may separate the sampled companies by random allocation. Random allocation has the advantage of eliminating the need to test for selection bias since the resulting estimation and holdout samples have the same composition structure. However, each of the latter samples contains both rated companies and newcomers. Such mixed composition does not agree with our prediction purpose.
} 
in consecutive nine years (April 1999 - April 2007) were classified into the estimation sample. The rest of the sampled companies were classified into the holdout sample. Based on the division principle, 413 companies were divided into the estimation sample and 366 companies into the holdout sample.

To examine whether our estimation and holdout samples induced selection bias, a procedure based on OLPM with sample selection was performed using LIMDEP 8.0 to test the null hypothesis of no selection bias caused by the above sample division principle. The result of the test shows no rejection of the null hypothesis of interest at $5 \%$ level of significance. Before performing the selection bias test, a forward selection procedure based on minimizing classification error rate on the estimation sample (Härdle et al., 2006) was used to objectively determine effective predictors for OLPM. The final list of the selected predictors includes industry effects, two market-driven variables, and two accounting variables measuring a firm's financial leverage and profitability. The values of estimated coefficients of the selected marketdriven and accounting variables all agree with their expected signs. This indicates that marketdriven variables and industry effects are also important to determine S\&P's LTRs. Our variable selection result coincides with that obtained by Hwang (2008) for predicting ratings in year 2005. On the other hand, to study the difference between the unsolicited and the solicited ratings, Poon (2003) suggested profitability and sovereign credit risk as two major factors in determining S\&P's LTRs. Furthermore, to assess biases in credit ratings assigned by Moody's and S\&P's for near-to-default issuers, Güttler and Wahrenburg (2007) used accounting and macroeconomic variables as major determinants of issuer credit ratings.

The remainder of this paper is organized as follows. In Section 2, our methodology for forecasting credit ratings based on OSPM is developed using concepts similar to those based on OLPM. In Section 3, we describe one real data set and provide some summary statistics. The summary statistics show that the predictors under consideration have reasonable power in discriminating the creditworthiness of companies. The real data set was analyzed using methods based on OLPM and OSPM. The prediction performance of each method was measured by the total error rate obtained from the holdout sample. By the error rates summarized in Section 3, we conclude that the prediction method based on OSPM has better performance, and has potential to be a powerful credit rating prediction method. Concluding remarks and future research topics are contained in Section 4. Our theoretical results are presented in Appendix A. Finally, sketches of the proofs are given in Appendix B.

\section{Methodology}

In this section, we first briefly review the formulation of OLPM. The detailed introduction of OLPM can be referred to Kaplan and Urwitz (1979) and the manuscript by Borooah (2002). 
Then we describe the basic idea of OSPM and develop the methodology for estimating unknown quantities of OSPM.

\subsection{OLPM}

The OLPM is defined by imposing a linear regression relationship between S\&P’s LTRs and predictor variables. It is developed using the estimation sample composed of observations $\left(Y_{i}, x_{i}, z_{i}\right)$, for $i=1, \cdots, n$. The value of $Y_{i}=j$ indicates that the S\&P's LTR of the $i$-th company belongs to the category $j$, where $j \in\{1, \cdots, m\}$ and $m \geq 2$. Here $m$ stands for the total number of categories among S\&P's LTRs. The larger the value of $Y_{i}$, the better the S\&P's LTR category of the $i$-th company. The values of $x_{i}$ and $z_{i}$ are collected on the $i$-th company from the $d \times 1$ continuous and $q \times 1$ discrete explanatory variables $X$ and $Z$, respectively. Our aim is to predict the S\&P's LTR category for a given company without S\&P's LTR.

Given the estimation sample, the OLPM is defined by

$$
\left\{\begin{array}{l}
Y_{i}^{*}=\xi+\rho x_{i}+\varphi z_{i}+\varepsilon_{i}, \\
Y_{i}=j, \quad \text { if } \delta_{j-1}<Y_{i}^{*} \leq \delta_{j}, \quad \text { for } j=1, \cdots, m .
\end{array}\right.
$$

Here $\xi, \rho$, and $\varphi$ are $1 \times 1,1 \times d$, and $1 \times q$ vectors of parameters, $Y_{i}^{*}$ latent variables relating to S\&P's LTR assessment, and $\varepsilon_{i}$ independent standard normal random variables. Also $\delta_{0}, \cdots, \delta_{m}$ are threshold parameters discretizing the real line into $m$ intervals, where the values of $\delta_{j}$ are of ascending order and satisfy the conditions $\delta_{0}=-\infty, \delta_{1}=0$, and $\delta_{m}=\infty$. Set $\delta=\left(\delta_{2}, \cdots, \delta_{m-1}\right)$.

Given the model (1), the values of $\xi, \rho, \varphi$, and $\delta$ can be simply estimated by the maximum likelihood method (McCullagh, 1980). Let $\hat{\xi}, \hat{\rho}, \hat{\varphi}$, and $\hat{\delta}$ be their maximum likelihood estimates obtained by maximizing the log-likelihood function

$$
\ell(\xi, \rho, \varphi, \delta)=\sum_{i=1}^{n} \sum_{j=1}^{m} I\left(Y_{i}=j\right) \ln \left(\Phi_{i, j}-\Phi_{i, j-1}\right)
$$

of the estimation sample, where $\Phi_{i, j}=\Phi\left(\delta_{j}-\xi-\rho x_{i}-\varphi z_{i}\right), \Phi(\bullet)$ is the cumulative distribution function of standard normal random variable, and $I(\bullet)$ stands for the indicator function. Using the maximum likelihood estimates $\hat{\xi}, \hat{\rho}, \hat{\varphi}$, and $\hat{\delta}$, by the model (1), the S\&P’s LTR category of a given company with predictor values $\left(x_{0}, z_{0}\right)$ is predicted by

$$
\hat{Y}_{\text {OLPM }}\left(x_{0}, z_{0}\right)=j, \quad \text { if } \hat{\delta}_{j-1}<\hat{\xi}+\hat{\rho} x_{0}+\hat{\varphi} z_{0} \leq \hat{\delta}_{j}, \quad \text { for some } j \in\{1, \cdots, m\} .
$$


McCullagh (1980) showed that the maximum likelihood estimates $\hat{\xi}, \hat{\rho}, \hat{\varphi}$, and $\hat{\delta}$ are consistent for $\xi, \rho, \varphi$, and $\delta$, respectively. Thus, the resulting predicted regression function value $\hat{\xi}+\hat{\rho} x_{0}+\hat{\varphi} z_{0}$ converges to the true regression function value $\xi+\rho x_{0}+\varphi z_{0}$. This result shows that OLPM is an efficient prediction model if its regression function is correctly specified.

Hwang et al. (2008) pointed out that the prediction rule $\hat{Y}_{\text {OLPM }}\left(x_{0}, z_{0}\right)$ in (2) is equivalent to basing on cutoff value $1 / 2$ :

$$
\hat{Y}_{\text {OLPM }}\left(x_{0}, z_{0}\right)=j, \quad \text { if } \hat{\Phi}_{0, j-1}<1 / 2 \leq \hat{\Phi}_{0, j}, \quad \text { for some } j \in\{1, \cdots, m\} \text {, }
$$

where $\hat{\Phi}_{0,0}=0, \quad \hat{\Phi}_{0, j}=\Phi\left(\hat{\delta}_{j}-\hat{\xi}-\hat{\rho} x_{0}-\hat{\varphi} z_{0}\right)$, for $j=1, \cdots, m-1$, and $\hat{\Phi}_{0, m}=1$. For improving the performance of the prediction rule based on OLPM, they followed the idea of Altman (1968), Ohlson (1980), and Begley et al. (1996), and suggested replacing cutoff value $1 / 2$ in (3) with some cutoff value $p \in[0,1]$. The resulting prediction rule is denoted by

$$
\hat{Y}_{\text {OLPM }}\left(x_{0}, z_{0}\right)=j \text {, if } \hat{\Phi}_{0, j-1}<p \leq \hat{\Phi}_{0, j}, \quad \text { for some } j \in\{1, \cdots, m\} .
$$

In order to decide a proper data-based cutoff value for $p$, usually one would use the estimation sample to evaluate the performance of the classification scheme. In doing so, there are three types of "in-sample” error rate occurred on the estimation sample:

type I error rate $\alpha_{i n}(p)=n^{-1} \sum_{i=1}^{n} I\left\{\hat{Y}_{\text {OLPM }}\left(x_{i}, z_{i}\right)>Y_{i}\right\}$

type II error rate $\beta_{\text {in }}(p)=n^{-1} \sum_{i=1}^{n} I\left\{\hat{Y}_{\text {OLPM }}\left(x_{i}, z_{i}\right)<Y_{i}\right\}$

total error rate $\gamma_{i n}(p)=\alpha_{i n}(p)+\beta_{\text {in }}(p)$.

Using the estimation sample, $\alpha_{i n}(p)$ is the rate of misclassifying a company to a higher rating category, and $\beta_{\text {in }}(p)$ the rate of misclassifying a company to a lower rating category. If the type I error would cause severe losses to investors, then it is important to control the magnitude of $\alpha_{i n}(p)$. Thus, a proper data-based cutoff value $\hat{p}_{\text {OLPM }}(u)$ for $p$ may be determined such that

$$
\gamma_{\text {in }}\left\{\hat{p}_{\text {OLPM }}(u)\right\}=\min _{\alpha_{\text {in }}(p) \leq u, p \in[0,1]} \gamma_{\text {in }}(p),
$$

for each $u \in[0,1]$. This approach is to control the magnitude of in-sample type I error rate $\alpha_{i n}(p)$ to be at most $u$, so that the in-sample total error rate $\gamma_{i n}(p)$ is minimal. On the other hand, if the type II error would cause severe losses to investors, then we may control the magnitude of $\beta_{\text {in }}(p)$ instead. In practice, the value of $u \in[0,1]$ is determined by investors. If there is no restriction on the magnitude of $\alpha_{i n}(p)$ and $\beta_{\text {in }}(p)$, then we simply take $u=1$. The 
suggested cutoff value of $p$ in Hwang et al. (2008) is $\hat{p}_{\text {OLPM }}(1)$. Using the prediction rule based on OLPM with our data-based cutoff value $\hat{p}_{\text {OLPM }}(u)$, the S\&P's LTR category of the given company with predictor values $\left(x_{0}, z_{0}\right)$ is predicted by

$$
\hat{Y}_{\text {OLPM }}\left(x_{0}, z_{0}\right)=j, \quad \text { if } \hat{\Phi}_{0, j-1}<\hat{p}_{\text {OLPM }}(u) \leq \hat{\Phi}_{0, j}, \quad \text { for some } j \in\{1, \cdots, m\},
$$

for each $u \in[0,1]$. The prediction rule $\hat{Y}_{\text {OLPM }}\left(x_{0}, z_{0}\right)$ in (6) will be used in Section 3 to analyze a real data example.

\subsection{OSPM}

By the nature of linear regression function, OLPM has advantages of simple computation and interpretation. However, if it uses an improper regression function, then it has a danger to reach erroneous prediction. This limitation of OLPM can be improved by removing the restriction that its regression function is linear. In this paper, we suggest an OSPM which is more flexible in modeling the regression function. The OSPM is constructed by replacing the linear regression function in OLPM with a semiparametric regression function. It is defined by

$$
\left\{\begin{array}{l}
Y_{i}^{*}=H\left(x_{i}\right)+\theta z_{i}+\varepsilon_{i}, \\
Y_{i}=j, \text { if } \tau_{j-1}<Y_{i}^{*} \leq \tau_{j}, \text { for } j=1, \cdots, m .
\end{array}\right.
$$

Here $\theta, \tau_{0}, \cdots, \tau_{m}$ are the same as $\varphi, \delta_{0}, \cdots, \delta_{m}$ in the model (1), respectively, and $H(x)$ is an unknown but smooth function of the value $x$ of the d-dimensional continuous explanatory variable $X$. Set $\tau=\left(\tau_{2}, \cdots, \tau_{m-1}\right)$.

Following the same development of $\ell(\xi, \rho, \varphi, \delta)$, the corresponding log-likelihood function of the estimation sample based on OSPM is expressed by

$$
\ell^{*}(H, \theta, \tau)=\sum_{i=1}^{n} \sum_{j=1}^{m} I\left(Y_{i}=j\right) \ln \left(\Phi_{i, j}^{*}-\Phi_{i, j-1}^{*}\right),
$$

where $\Phi_{i, j}^{*}=\Phi\left\{\tau_{j}-H\left(x_{i}\right)-\theta z_{i}\right\}$. For a given company with predictor values $\left(x_{0}, z_{0}\right)$, if $H\left(x_{0}\right), \theta$, and $\tau$ can be efficiently estimated by $\hat{H}\left(x_{0}\right), \hat{\theta}$, and $\hat{\tau}$, then, by the model (7), its S\&P’s LTR category can be predicted by

$$
\hat{Y}_{\text {OSPM }}\left(x_{0}, z_{0}\right)=j \text {, if } \hat{\tau}_{j-1}<\hat{H}\left(x_{0}\right)+\hat{\theta} z_{0} \leq \hat{\tau}_{j}, \quad \text { for some } j \in\{1, \cdots, m\} .
$$

The same developments (3)-(6) can be applied to (8) for OSPM. Let $\hat{p}_{\text {OSPM }}(u)$ be similarly defined. Thus the prediction rule $\hat{Y}_{\text {OSPM }}\left(x_{0}, z_{0}\right)$ in (8) can be improved as

$$
\hat{Y}_{\text {OSPM }}\left(x_{0}, z_{0}\right)=j \text {, if } \hat{\Phi}_{0, j-1}^{*}<\hat{p}_{\text {OSPM }}(u) \leq \hat{\Phi}_{0, j}^{*} \text {, for some } j \in\{1, \cdots, m\} \text {, }
$$


for each $u \in[0,1]$, where $\hat{\Phi}_{0,0}^{*}=0, \quad \hat{\Phi}_{0, j}^{*}=\Phi\left\{\hat{\tau}_{j}-\hat{H}\left(x_{0}\right)-\hat{\theta} z_{0}\right\}$, for $j=1, \cdots, m-1$, and $\hat{\Phi}_{0, m}^{*}=1$. The prediction rule $\hat{Y}_{\text {OSPM }}\left(x_{0}, z_{0}\right)$ in (9) will be used in Section 3 to analyze a real data example. A local likelihood method will be employed in Sections 2.3 and 2.4 to estimate unknown parameters $H\left(x_{0}\right), \theta$, and $\tau$ of OSPM.

\subsection{A local likelihood method for estimating parameters in OSPM}

We now apply the local likelihood concept (Tibshirani and Hastie, 1987; Staniswallis, 1989; Fan et al., 1995; Hwang et al., 2007) to develop a procedure for estimating $H\left(x_{0}\right), \theta$, and $\tau$ in OSPM, where $x_{0}=\left(x_{01}, \cdots, x_{0 d}\right)^{T}$ is any given value of the d-dimensional continuous explanatory variable $X$. The basic idea of the local likelihood method is to center the data around $x_{0}$, and weight the log-likelihood such that it places more emphasis on those observations nearest to $x_{0}$. It can be simply performed by first considering a neighborhood $S_{b}\left(x_{0}\right)=\left\{u=\left(u_{1}, \cdots, u_{d}\right)^{T}:\left|u_{k}-x_{0 k}\right| \leq b\right.$, for each $\left.k=1, \cdots, d\right\}$ of $x_{0}$. Here $b$ is some positive constant to be determined later by the estimation sample, and called the bandwidth. If the value of $b$ is small enough and $x_{i}$ belongs to $S_{b}\left(x_{0}\right)$, then Taylor's first order expansion says that $H\left(x_{i}\right) \approx H\left(x_{0}\right)+H^{(1)}\left(x_{0}\right)^{T}\left(x_{i}-x_{0}\right)$. This result means that such $H\left(x_{i}\right)$ in $\ell^{*}(H, \theta, \tau)$ can be approximated by $\eta_{0}+\eta_{1}\left(x_{i}-x_{0}\right)$, where $\eta_{0}$ is a scalar parameter representing $H\left(x_{0}\right)$ and $\eta_{1}$ a $1 \times d$ vector of parameters standing for $H^{(1)}\left(x_{0}\right)^{T}$. Set $\eta=\left(\eta_{0}, \eta_{1}\right)$

Based on the above discussion, a local likelihood method for making inference about $(\eta, \theta, \tau)$ can be proposed by modifying $\ell^{*}(H, \theta, \tau)$ to consider the following "local (weighted)" log-likelihood function

$$
\ell_{0}^{*}\left(\eta, \theta, \tau ; x_{0}\right)=\sum_{i=1}^{n} \sum_{j=1}^{m} I\left(Y_{i}=j\right) \ln \left(\Phi_{i, j}^{\circ}-\Phi_{i, j-1}^{\circ}\right) W\left(x_{i}\right),
$$

where $\Phi_{i, j}^{\circ}=\Phi\left\{\tau_{j}-\eta_{0}-\eta_{1}\left(x_{i}-x_{0}\right)-\theta z_{i}\right\}$. The simplest weight $W\left(x_{i}\right)$ assigned to the observation $\left(Y_{i}, x_{i}, z_{i}\right)$ in $\ell_{0}^{*}\left(\eta, \theta, \tau ; x_{0}\right)$ is the indicator value $I\left\{x_{i} \in S_{b}\left(x_{0}\right)\right\}$. In this case, it can be mathematically defined as $W\left(x_{i}\right)=K_{b}\left(x_{i}-x_{0}\right)=b^{-d} \prod_{k=1}^{d} K\left\{\left(x_{i k}-x_{0 k}\right) / b\right\}$, where $K$ is the uniform probability density function over $[-1,1]$, also called the kernel function, and $x_{i}=\left(x_{i 1}, \cdots, x_{i d}\right)^{T}$. 
Conceptually, a more general weighting scheme can be used for defining weights $W\left(x_{i}\right)$ when constructing the local log-likelihood function. This can be achieved by taking $K$ as a symmetric and unimodal probability density function supported on the interval $[-1,1]$. The results in the literature showed that the choice of bandwidth $b$ plays important role, but the choice of kernel function $K$ is not very important in the weighting scheme. Some discussions of the kernel weighting method can be found in the monographs by Eubank (1988), Müller (1988), Härdle (1990, 1991), Scott (1992), Wand and Jones (1995), Fan and Gijbels (1996), and Simonoff (1996). In this paper, we select $W\left(x_{i}\right)=K_{b}\left(x_{i}-x_{0}\right)$ with a general $K$ in all analyses.

Set $(\tilde{\eta}, \tilde{\theta}, \tilde{\tau})$ as the maximizer of $\ell_{0}^{*}\left(\eta, \theta, \tau ; x_{0}\right)$, where $\tilde{\eta}=\left(\tilde{\eta}_{0}, \tilde{\eta}_{1}\right)$. We define $\tilde{H}\left(x_{0}\right)=\tilde{\eta}_{0}$ to indicate that it is an estimate of $H\left(x_{0}\right)$. We also point out that $\theta$ and $\tau$ are global parameters and their corresponding estimates $\tilde{\theta}$ and $\tilde{\tau}$ produced from $\ell_{0}^{*}\left(\eta, \theta, \tau ; x_{0}\right)$ may not be efficient, since such estimates are derived by maximizing a local log-likelihood function depending on $x_{0}$. In Section 2.4, more efficient estimates of $H\left(x_{0}\right), \theta$, and $\tau$ can be achieved.

\subsection{More efficient estimates of parameters in OSPM}

More efficient estimates of $H\left(x_{0}\right), \theta$, and $\tau$ can be derived using the following two-step procedure. We first note that, for each value $x_{i}$, an initial estimate $\tilde{H}\left(x_{i}\right)$ of $H\left(x_{i}\right)$ can be obtained by the method outlined in Section 2.3. The two-step procedure includes:

Step 1: $\theta$ and $\tau$ are estimated by maximizing the pseudo log-likelihood function

$$
\ell_{1}^{*}(\theta, \tau)=\sum_{i=1}^{n} \sum_{j=1}^{m} I\left(Y_{i}=j\right) \ln \left(\tilde{\Phi}_{i, j}-\widetilde{\Phi}_{i, j-1}\right),
$$

where $\widetilde{\Phi}_{i, j}=\Phi\left\{\tau_{j}-\tilde{H}\left(x_{i}\right)-\theta z_{i}\right\}$. Here $\ell_{1}^{*}(\theta, \tau)$ is obtained by replacing each $H\left(x_{i}\right)$ in $\ell^{*}(H, \theta, \tau)$ with its initial estimate $\tilde{H}\left(x_{i}\right)$. Set $(\hat{\theta}, \hat{\tau})$ as the maximizer of $\ell_{1}^{*}(\theta, \tau)$. The estimates of $(\theta, \tau)$ are taken as $(\hat{\theta}, \hat{\tau})$.

Step 2: $H\left(x_{0}\right)$ is estimated by maximizing the pseudo local log-likelihood function

$$
\ell_{2}^{*}\left(\eta ; x_{0}\right)=\sum_{i=1}^{n} \sum_{j=1}^{m} I\left(Y_{i}=j\right) \ln \left(\hat{\Phi}_{i, j}^{\circ}-\hat{\Phi}_{i, j-1}^{\circ}\right) K_{g}\left(x_{i}-x_{0}\right),
$$


where $\hat{\Phi}_{i, j}^{\circ}=\Phi\left\{\hat{\tau}_{j}-\eta_{0}-\eta_{1}\left(x_{i}-x_{0}\right)-\hat{\theta} z_{i}\right\}$. Here $\ell_{2}^{*}\left(\eta ; x_{0}\right)$ is obtained by replacing $\theta$ and $\tau$ in $\ell_{0}^{*}\left(\eta, \theta, \tau ; x_{0}\right)$ with their estimates $\hat{\theta}$ and $\hat{\tau}$ produced in Step 1 . Set $\hat{\eta}=\left(\hat{\eta}_{0}, \hat{\eta}_{1}\right)$ as the maximizer of $\ell_{2}^{*}\left(\eta ; x_{0}\right)$. The estimate of $H\left(x_{0}\right)$ is given by $\hat{H}\left(x_{0}\right)=\hat{\eta}_{0}$.

Note that in Step 2 we have used a different bandwidth $g$ in the local likelihood method. We allow $b$ and $g$ to be different in the analysis but emphasize that both values will be determined by the estimation sample (see our proposal given in Section 2.5). We suggest that the final estimates of $H\left(x_{0}\right), \theta$, and $\tau$ be defined by $\hat{H}\left(x_{0}\right), \hat{\theta}$, and $\hat{\tau}$, respectively. Their theoretical properties will be given in Appendix A.

2.5. Choosing the kernel function $K$ and the values of $(b, g, p)$

Our Theorem 1 in Appendix A shows that $\hat{H}\left(x_{0}\right), \hat{\theta}$, and $\hat{\tau}$ are consistent estimators of $H\left(x_{0}\right), \theta$, and $\tau$, respectively. This result means that the prediction rule $\hat{Y}_{O S P M}\left(x_{0}, z_{0}\right)$ in (9) is reliable. To compute its value, we first need to choose the kernel function $K$ and the values of $(b, g, p)$. Remark 1 in Appendix A points out that, in the sense of yielding smaller asymptotic mean integrated square error of $\hat{H}\left(x_{0}\right)$, the optimal $K$ is the Epanechnikov kernel defined as $K(t)=(3 / 4)\left(1-t^{2}\right) I(|t| \leq 1)$, and the optimal value of $g$ is of larger order than that of $b$. Thus our kernel function $K$ in applications is taken as the Epanechnikov kernel. But the optimal values of $(b, g)$ are not available in practice for depending on the unknown factors $H(x), \theta$, and $\tau$.

To choose the values of $(b, g, p)$, we suggest considering the in-sample type I, type II, and total error rates of the classification scheme based on OSPM in (9) as functions of $(b, g, p)$, denoted as $\alpha_{i n}(b, g, p), \quad \beta_{i n}(b, g, p)$, and $\gamma_{i n}(b, g, p)$, respectively. The definition of insample error rates has been given in Section 2.1. The proper values for $(b, g, p)$ are then simultaneously determined so that $\gamma_{i n}(b, g, p)$ is minimal, subject to the constraints $p \in[0,1]$, $0<b<g$, and $\alpha_{i n}(b, g, p) \leq u$, for each $u \in[0,1]$. Such selected values for $(b, g, p)$ are denoted as $\left\{\hat{b}(u), \hat{g}(u), \hat{p}_{\text {OSPM }}(u)\right\}$. 
The performance of the prediction rule based on OSPM in (9) is measured by the "out-ofsample" error rates. These error rates are evaluated on the holdout sample. Suppose that the holdout sample is composed of observations $\left(\tilde{Y}_{i}, \tilde{X}_{i}, \tilde{z}_{i}\right)$, for $i=1, \cdots, n_{0}$. Using the estimation sample, the Epanechnikov kernel, and the selected values $\left\{\hat{b}(u), \hat{g}(u), \hat{p}_{\text {OSPM }}(u)\right\}$, the value of $\hat{H}\left(\tilde{x}_{i}\right)$, for each data point $\left(\tilde{x}_{i}, \tilde{z}_{i}\right)$ in the holdout sample and those of $\hat{\theta}$ and $\hat{\tau}$ can be computed. The S\&P's LTR category for a company with predictor values $\left(\tilde{x}_{i}, \tilde{z}_{i}\right)$ in the holdout sample is predicted by $\hat{Y}_{\text {OSPM }}\left(\tilde{X}_{i}, \tilde{z}_{i}\right)$ as defined in (9). After the evaluation procedure is completed for each company in the holdout sample, the out-of-sample error rates for the prediction rule based on OSPM are defined by

$$
\begin{aligned}
& \text { type I error rate } \alpha_{\text {out }}(u)=n_{0}^{-1} \sum_{i=1}^{n_{0}} I\left\{\hat{Y}_{\text {OSPM }}\left(\tilde{x}_{i}, \tilde{z}_{i}\right)>\tilde{Y}_{i}\right\}, \\
& \text { type II error rate } \beta_{\text {out }}(u)=n_{0}^{-1} \sum_{i=1}^{n_{0}} I\left\{\hat{Y}_{\text {OSPM }}\left(\tilde{x}_{i}, \tilde{z}_{i}\right)<\tilde{Y}_{i}\right\}, \\
& \text { total error rate } \gamma_{\text {out }}(u)=\alpha_{\text {out }}(u)+\beta_{\text {out }}(u),
\end{aligned}
$$

for each $u \in[0,1]$. Given the holdout sample, the out-of-sample error rates can be similarly defined for the prediction rule based on OLPM in (6).

\section{An empirical study}

In this section, an empirical study was performed to investigate the performance of the two prediction rules in (6) and (9) based on OLPM and OSPM, respectively.

\subsection{The data}

Each sampled company must: (i) be listed on the New York Stock Exchange, American Stock Exchange, or NASDAQ, (ii) adopt calendar fiscal year, (iii) not be a financial services company with the SIC code 6000-6999, (iv) have a S\&P’s LTR in April 2007, and (v) have complete values of the twenty-four potential predictors for studying S\&P’s LTRs in April 2007. The criterion (i) guarantees that market-driven variables are available. The criterion (ii) synchronizes the timing of predictors in the sense that all market-driven and accounting variables cover the same calendar year. The criterion (iii) excludes the financial services companies since they are subject to regulations and adopt different accounting conventions. The criterion (iv) 
makes sure that the S\&P's LTR is available. Finally, the criterion (v) excludes the companies with incomplete potential predictor values. The data were collected from both COMPUSTAT and CRSP databases. Since S\&P's considers predictor values as their three-year averages, this study follows the same method (Blume et al., 1998; Poon, 2003). Thus, the value of each of the twenty-four potential predictors for studying S\&P's LTRs in April 2007 was taken as the average of its values available from the two databases in years 2004, 2005, and 2006.

Based on the COMPUSTAT database, there were 3785 companies satisfying the selection criteria (i)-(iii), but only 918 companies among them receiving S\&P’s LTRs in April 2007. However, among those 918 companies, there were 784 companies having complete values of the twenty-four potential predictors for studying S\&P's LTRs in April 2007. The missing data problem is not unusual in applications, especially when there are many predictors in the model. However, as long as the missingness occurs at random then the sample will not introduce systematic bias in our analyses (Allison, 2001; Little and Rubin, 2002). We have no reason not to believe that the missingness occurred in COMPUSTAT and CRSP databases is missing at random. On the other hand, in order to study industry effects on S\&P's LTRs, the industries were classified by the first digit in the four-digit SIC code. Among the 784 companies, there were only three companies having SIC code less than 1000 and two companies carrying SIC code greater than 9000. These five companies were dropped from the sample. ${ }^{3}$ Thus our final sample consisted of 779 companies.

For purpose of later analysis, the final sample was further divided into the estimation sample and the holdout sample based on the longevity of S\&P's LTR. The reason for adopting this division principle has been given in footnote 2 in Section 1. According to S\&P's Research Insight North America Data Guide, S\&P’s began using the term LTR on September 1, 1998. Companies receiving S\&P’s LTRs in consecutive nine years (April 1999 - April 2007) were classified into the estimation sample. The rest of the sampled companies were classified into the holdout sample. Among our 779 sampled companies, there were 413 and 366 estimation and holdout companies, respectively. Table 1 presents the frequency distributions of the estimation and holdout companies according to different S\&P's LTR categories and SIC codes.

S\&P's LTR ranges from AAA to D. Panel A of Table 1 presents the frequency distribution of the sampled companies according to their S\&P's LTRs. Based on the result from the estimation sample, it seems reasonable to group S\&P’s LTRs into three categories: $\{$ Below BBB

\footnotetext{
${ }^{3}$ If such industries with few companies are included in the prediction model, then the resulting number of industry indicator variables is increased, thus the estimates of parameters in the corresponding model might become less precise.
} 
as category $1,\{\mathrm{BBB}\}$ as category 2 , and $\{\mathrm{AAA}, \mathrm{AA}, \mathrm{A}\}$ as category $3 .^{4}$ According to S\&P’s opinion, firms in the $\{\mathrm{AAA}, \mathrm{AA}, \mathrm{A}\}$ category mean that they have demonstrated strong capacity to meet their financial obligations. Firms receiving BBB rating mean that they have adequate capacity to meet their financial commitments. However, firms receiving LTR below BBB mean that they are regarded as having speculative characteristics. Panel B of Table 1 gives the frequency distribution of the sampled companies according to the three S\&P's LTR categories. Note that the companies in our holdout sample are newcomers in the pool of companies with S\&P's LTRs, in contrast to those in the estimation sample. Panel B of Table 1 shows that the holdout companies have lower S\&P's LTRs on the average. Such result agrees with the observation reported in Pettit et al. (2004) and Hwang et al. (2008). Blume et al. (1998) also reported the similar observation for bond ratings. The distribution of the companies in the holdout sample shows that there are about $68.6 \%$ companies in the speculative S\&P's LTR category. In contrast, there are only about $35.6 \%$ companies in the estimation sample with the speculative S\&P's LTR category.

The twenty-four potential explanatory variables for studying S\&P's LTRs in year 2007 include four market-driven variables, nineteen accounting variables, and industry effects. Their definitions are given in Table 2. The four market-driven variables are excess return (EXRET), relative size (RSIZE), standard deviation of monthly returns (SIGMA), and KMV-Merton default probability (KMV). ${ }^{5}$ The nineteen accounting variables measure different aspects (size, financial leverage, coverage, cash flow, profitability, and liquidity) of financial health of a company. The industry effects were estimated through the coefficients of six industry indicator variables in the model. Using the data in the estimation sample, Table 3 shows summary statistics and F-tests of equality of the means among the three S\&P's LTR categories for the market-driven variables and accounting variables. The $p$-values in Table 3 show that testing the null hypothesis of equal means is significant at 0.05 level for each of the four market-driven variables and are significant for all accounting variables, except EM, QR, and CASHR. This result indicates that most of the variables considered in this paper are effective predictive variables. Table 3 also shows that, on the average, if a company has larger firm size, smaller financial leverage, larger coverage, larger cash flow, or larger profit, then it has better S\&P's LTR category.

\footnotetext{
${ }^{4}$ The estimation sample was divided into the three categories so that the resulting three cells have approximately equal sizes. On the other hand, if one divides the estimation sample into more categories, then some cells have smaller sizes and the number of threshold parameters increases. Thus the resulting estimates of parameters in the corresponding model might become less precise.

${ }^{5}$ Since the computation of KMV-Merton default probability requires the market value of equity, it is treated as a market-driven variable. Its detailed computational procedure can be referred to Bharath and Shumway (2008).
} 


\subsection{Testing selection bias}

To examine whether our criterion for dividing the overall sample into estimation and holdout samples induced selection bias, the procedure of OLPM under our particular sample selection was performed. This procedure was designed by simultaneously applying OLPM to the estimation sample, and applying the two-class linear probit model to the particular sample in which the classes 0 and 1 were assigned respectively to the holdout and estimation companies (see Model 1 of Table 5). The detail of this approach can be found in Greene (2002). Before performing it, a forward selection procedure based on minimizing in-sample total error rate was first used to objectively determine effective predictors for OLPM. The in-sample total error rate was computed using the classification scheme based on OLPM in (3) for simplicity. The variable selection procedure asked that the newly entered variable must be able to reduce at least one percent of in-sample total error rate of the classification scheme using those already entered variables. Table 4 gives the variable selection result. It shows that the final list of the selected predictors in OLPM includes LDC, ROE, RSIZE, SIGMA, and industry effects. Results in Table 3, by using F-test for testing equality of three means (corresponding to the three S\&P's LTR categories), show that the last four selected continuous predictors are all significant at the $1 \%$ level.

Using the five selected variables, Table 5 shows the results obtained from performing the procedure of OLPM, under our particular sample selection, based on the application of LIMDEP 8.0. Panel C of Model 1 in Table 5 shows that the null hypothesis of corr $=0$ was not rejected at $5 \%$ level of significance. Here the null hypothesis of $\operatorname{corr}=0$ stands for no sample selection bias caused by our criterion of dividing the overall sample. On the other hand, Model 2 in Table 5 shows the results of OLPM, including $p$-value of the chi-squared test for model fit. It is also of interest to note that by comparing the parameter estimates of the two OLPMs (in Models 1 and 2 of Table 5), we find out that their values are approximately equal. Thus since there is no sample selection bias, the results of Model 2 are adopted for predicting S\&P's LTR categories for companies in the holdout sample.

It is important to note that the two selected predictors SIGMA and LDC measure various aspects of risk and financial leverage of an issuer, respectively. The larger the values of these two predictors (SIGMA and LDC), the lower the S\&P's LTR category. That is, these two selected predictors should be negatively correlated with rating, and the signs of their coefficients should be negative. Another two selected predictors RSIZE and ROE stand for the market capitalization and profitability of an issuer, respectively. The larger the values of these two predictors, the better the creditworthiness of an issuer. This implies that RSIZE and ROE should be positively correlated with rating, and the signs of their coefficients should be positive. From 
Model 2 of Table 5, the signs of the values of estimated coefficients for these four selected predictors LDC, ROE, RSIZE, and SIGMA all agree with our expectation. Model 2 also shows that not all industries have the same effect on S\&P's LTRs, thus the industry effects are useful for predicting ratings.

\subsection{Computational procedures}

In computing OSPM, the values of the four selected continuous predictors LDC, ROE, RSIZE, and SIGMA were first divided by their sample standard deviations so that they have the same scale. This is important, since the influence of the predictor with very large range in estimating the optimal values of $(b, g, p)$ can be avoided. The kernel function $K$ was taken as the Epanechnikov kernel.

A grid-search approach was used in computing the optimal values of $(b, g, p)$ for OSPM. The values of $\gamma_{i n}(b, g, p)$ on the equally spaced logarithmic grid of $51 \times 51 \times 1001$ values of $(b, g, p)$ in $[0.5,3] \times[0.5,3] \times\left[10^{-5}, 1\right]$ were computed. See Marron and Wand (1992) for a discussion that an equally spaced grid of parameters is typically not a very efficient design for this type of grid search. Given each value of $u \in[0,1]$, the global minimizer $\left\{\hat{b}(u), \hat{g}(u), \hat{p}_{\text {OSPM }}(u)\right\}$ of $\gamma_{\text {in }}(b, g, p)$ on the grid points with restrictions $p \in[0,1]$, $0<b<g$, and $\alpha_{i n}(b, g, p) \leq u$ was taken as the optimal values of $(b, g, p)$.

Using the estimation sample and the values of $\left\{\hat{b}(1), \hat{g}(1), \hat{p}_{\text {OSPM }}(1)\right\}$, Figure 1 shows the plot of $\left\{x_{j}, \hat{H}(x)\right\}$ produced from OSPM for each of the four selected continuous predictors LDC, ROE, RSIZE, and SIGMA. Here $x_{j}$ stands for the value of the $j$-th selected continuous predictor, and $x$ has the $j$-th component as $x_{j}$, but all other components are fixed at their sample median levels. In the plot of $\left\{x_{j}, \hat{H}(x)\right\}$, we have taken the left and the right boundary points of its horizontal axe as the 0.5 and 99.5 percentiles of the values of the $j$-th selected continuous predictor, respectively. These plots are used to visually check the adequacy of the order-one polynomial function assumed for each continuous predictor in the linear regression function of OLPM. From panels (a)-(d) of Figure 1, the slope of each curve agrees with the expected direction of the corresponding variable effect. However, we also see that the order-one polynomial assumed for each continuous predictor by OLPM is inadequate, since it is clear that there is a nonlinear relationship between $x_{j}$ and $\hat{H}(x)$ for each of ROE, RSIZE, and SIGMA, except LDC. 


\subsection{Prediction results}

Given the estimation and the holdout samples, the performance of the two discussed prediction rules based on OLPM and OSPM are presented in Figure 2 and Tables 6 and 7.

Figure 2 shows the error rates of the two discussed prediction rules. These error rates were derived under the constraint that the in-sample type I error rate was at most $u$. Panels (a), (c), and (e) of Figure 2 show the in-sample error rates of the two prediction rules. In the case of $u \leq 0.08$, their in-sample type I error rates are close to the designed bounds, but the in-sample type II and total error rates of OSPM are smaller than those of OLPM. The largest percentage decrease of the in-sample total error rate by OSPM over OLPM is about 21\%. On the other hand, panels (b), (d), and (f) of Figure 2 show the out-of-sample error rates of the two prediction rules. Their out-of-sample type II error rates are very similar, for all $u \in[0,1]$. However, the out-ofsample type I and total error rates of OSPM are in general smaller than those of OLPM, for all $u \in[0,1]$. The largest percentage decrease of the out-of-sample total error rate by OSPM over OLPM is $22 \%$. Considering the out-of-sample type I and total error rates, OSPM clearly outperforms OLPM, for all $u \in[0,1]$.

In the case of $u=1$, the results of classifying the estimation companies and predicting the holdout companies are given in Tables 6 and 7, respectively. All error rates shown in these two tables were produced under no constraint on the magnitude of in-sample type I or type II error rate. Table 6 shows that OSPM has smaller in-sample type I and total error rates, but larger insample type II error rate. Table 7 shows that OSPM has better prediction performance than OLPM, since the out-of-sample total error rates of their predictions are equal to $18.9 \%$ and $24.0 \%$, respectively. Also, OSPM has better ability in predicting the speculative grade \{Below BBB\}. This is important, since misclassifying speculative grade to investment grade ( $\{\mathrm{BBB}\}$ or $\{\mathrm{AAA}$, AA, A \}) might cause severe losses to investors.

\section{Conclusion remarks and future research topics}

In this paper, multiple-class prediction methods based on OSPM is proposed. Our OSPM is developed by replacing the linear regression function $\xi+\rho x+\varphi z$ of OLPM with a semiparametric regression function $H(x)+\theta z$. Here $H(x)$ is an unknown but smooth function of the value $x$ of the d-dimensional continuous explanatory variable $X$, and $z$ is the value of $q \times 1$ discrete explanatory variable $Z$. Hence OSPM is more flexible than OLPM in modeling the regression function. The estimators of unknown quantities in OSPM are developed from the local likelihood method, and computed by maximizing a weighted log-likelihood function. Thus 
the required computation for OSPM is as simple as that for OLPM. The large sample properties of these estimators are studied through their asymptotic bias and variance. Theoretical results show that the computed regression function value using OSPM consistently estimates the true regression function value. Thus OSPM is a reliable prediction model.

To decide the optimal prediction rule, we propose to control the magnitude of in-sample type I error rate to be at most $u$, so that the sum of in-sample type I and II error rates is minimal. Based on the estimation sample, the type I error rate denotes the rate of misclassifying a company to a higher rating category, and type II error rate stands for the rate of misclassifying a company to a lower rating category. Controlling the magnitude of type I error rate is important, since the type I error might cause severe losses to investors.

One additional advantage of using OSPM is that the relation between $H(x)$ and the $\mathrm{d}$ dimensional predictive variable $x$ can be obtained from the plots of $\left\{x_{j}, \hat{H}(x)\right\}$, for $j=1, \cdots, d$. Here $x_{j}$ denotes the value of the $j$-th continuous predictor, and $x$ in $\hat{H}(x)$ has the $j$-th component as $x_{j}$, but all other components are fixed at some values, for example their sample medians. Using these plots of $\left\{x_{j}, \hat{H}(x)\right\}$, the adequacy of the order-one polynomial function assumed for each continuous predictor in the linear regression function of OLPM can be visually checked. If the linear regression function of OLPM is not proper, the plots of $\left\{x_{j}, \hat{H}(x)\right\}$ may guide us on how to make a better selection of parametric regression function for ordered probit model. For example, if the plot of $\left\{x_{j}, \hat{H}(x)\right\}$, for some $j$, presents a quadratic relation, then the relation between the regression function and $x_{j}$ should be an order-two polynomial. Sometime, using a parametric regression function in ordered probit model is important, particularly when one has many predictors to be considered simultaneously and does not have enough sample data to estimate the regression function nonparametrically.

One real data example for predicting S\&P’s LTRs in year 2007 has been used to illustrate OSPM. To find important predictors of S\&P’s LTRs, we have considered twenty-four potential predictors used in previous studies. The twenty-four potential predictors include four marketdriven variables, nineteen accounting variables, and industry effects. A data set containing 779 companies (413 estimation companies and 366 holdout companies) having complete values of the twenty-four potential predictors for studying S\&P’s LTRs in April 2007 was collected from COMPUSTAT and CRSP databases. The results obtained by the forward selection procedure show that the final list of the selected predictors for OLPM contains industry effects, two marketdriven variables, and two accounting variables. Given the estimation and the holdout samples, our empirical results demonstrate that the prediction rule based on OSPM has better performance 
than that based on OLPM, in the sense of having smaller out-of-sample total error rate.

In order to estimate OSPM in practice, we need to decide proper values of bandwidth parameters $b$ and $g$. In this paper, we suggest using a grid search approach to find those proper values. However, this approach suffers from heavy computational burden. One possible remedy for this drawback is to use the plug-in method to estimate those proper values. For example, we may determine those values by minimizing the estimated mean square error of each estimator $\hat{H}(x)$ and $\hat{\theta}$. For more discussion of the plug-in approach, see for example Härdle et al. (1992) and Jones et al. (1996).

There are some possible extensions for the methods considered in this paper. First, in this paper, we only used cross-sectional data to study the performance of OSPM. The model can also be applied to panel data with independence assumption (Blume et al., 1998; Poon, 2003; Güttler and Wahrenburg, 2007). Secondly, to account for the autocorrelations among panel data, we may introduce a dynamic OLPM or OSPM with autocorrelation structure (Lipsitz et al., 1994; Müller and Czado, 2005) to study credit ratings. Thirdly, the performance of OSPM for predicting credit ratings was only studied in this paper using firm-specific variables including market-driven variables, accounting variables, and industry effects. The macroeconomic variables such as change in GDP and unemployment rate have been considered in Güttler and Wahrenburg (2007) as major determinants of credit ratings. It is of interest to study the effects of macroeconomic variables on our OSPM for predicting credit ratings in the future research. Finally, the industry effects on S\&P's LTRs were studied by introducing industry indicator variables in each of OLPM and OSPM. They could also be studied using frailty factors to describe the unobservable heterogeneity (Duffie et al., 2006; Chava at al., 2008).

\section{Appendix A. Theoretical Results}

In Appendix A, we shall present asymptotic properties of estimators $\hat{\theta}, \hat{\tau}$, and $\hat{H}\left(x_{0}\right)$. Those of $\tilde{H}\left(x_{0}\right)$ are shown in (A10) of Appendix B. The composition of the estimation sample and the formulation of these estimators have been given in Section 2.1 and in Sections 2.3-2.4, respectively.

To study asymptotic properties of estimators $\hat{\theta}, \hat{\tau}, \tilde{H}\left(x_{0}\right)$, and $\hat{H}\left(x_{0}\right)$, we need the following conditions:

(C1) The function $H(x)$ is defined on $[0,1]^{d}$, and each of its second order partial derivative is Lipschitz continuous on $[0,1]^{d}$. 
(C2) The frequency function $f(x, z)$ of $(X, Z)$ is Lipschitz continuous and bounded above zero on $[0,1]^{d}$ with respect to $x$, for each $z$. Also, the conditional probability density function $f(z \mid x)$ of $Z$ given $X=x$ can not be zero or one for each $x$, and is Lipschitz continuous with respect to $x$.

(C3) The kernel function $K$ is a symmetric and Lipschitz continuous probability density function supported on $[-1,1]$.

(C4) The values of $b$ and $g$ are selected on the interval $\left[s n^{-1+s}, s^{-1} n^{-s}\right]$, where $s$ is an arbitrarily small positive constant. The values of $b$ and $g$ satisfy $n b^{d+2}>>1>>g>>b$. The notation $a_{n}>b_{n}$ means that $b_{n} / a_{n} \rightarrow 0$, as $n \rightarrow \infty$.

Conditions (C1)-(C4) are regular for the usual nonparametric regression analysis. The bounded region $[0,1]^{d}$ in (C1) and (C2) is given for simplicity of presentation. The first part of condition (C2) guarantees that in estimating $H\left(x_{0}\right)$, for each $x_{0}=\left(x_{01}, \cdots, x_{0 d}\right) \in[0,1]^{d}$, the probability of $X \in \prod_{j=1}^{d}\left[x_{0 j}-b, x_{0 j}+b\right]$ is not zero. The second part of (C2) makes sure that Hessian matrices of log-likelihood functions $\ell_{0}^{*}\left(\eta, \theta, \tau ; x_{0}\right)$ and $\ell_{2}^{*}\left(\eta ; x_{0}\right)$ given in Sections 2.32.4 are invertible.

In order to give concise expressions for asymptotic properties of estimators $\hat{\theta}, \hat{\tau}$, and $\hat{H}\left(x_{0}\right)$, we need more notation. Let $H^{(2)}(u)=\left[H_{i, j}(u)\right]_{d \times d}$ be the Hessian matrix of the dvariate function $H$ at $u=\left(u_{1}, \cdots, u_{d}\right)^{T}, K^{\#}(u)=\prod_{j=1}^{d} K\left(u_{j}\right), \varphi_{k}=\max \left\{-1,\left(x_{0 k}-1\right) / b\right\}$, and $\rho_{k}=\min \left\{1, x_{0 k} / b\right\}$, for $k=1, \cdots, d$. Define

$$
\begin{gathered}
\kappa_{0}=\int_{\varphi_{1}}^{\rho_{1}} \cdots \int_{\varphi_{d}}^{\rho_{d}} K^{\#}(u) d u, \quad \kappa_{1}=\int_{\varphi_{1}}^{\rho_{1}} \cdots \int_{\varphi_{d}}^{\rho_{d}} u K^{\#}(u) d u, \quad \kappa_{2}=\int_{\varphi_{1}}^{\rho_{1}} \cdots \int_{\varphi_{d}}^{\rho_{d}}\left(u u^{T}\right) K^{\#}(u) d u, \\
\kappa_{2}^{*}=\int_{\varphi_{1}}^{\rho_{1}} \cdots \int_{\varphi_{d}}^{\rho_{d}}\left\{u^{T} H^{(2)}\left(x_{0}\right) u\right\} K^{\#}(u) d u, \quad \kappa_{3}^{*}=\int_{\varphi_{1}}^{\rho_{1}} \cdots \int_{\varphi_{d}}^{\rho_{d}} u\left\{u^{T} H^{(2)}\left(x_{0}\right) u\right\} K^{\#}(u) d u .
\end{gathered}
$$

Set $Q$ as the collection of all values of the discrete q-dimensional explanatory variable $Z$, $d_{j}(u, z)=\phi_{j}(u, z)-\phi_{j-1}(u, z), \quad$ and $\quad \psi_{j}(u, z)=\left\{\phi_{j}(u, z) e_{j}-\phi_{j-1}(u, z) e_{j-1}\right\} / d_{j}(u, z), \quad$ for $j=1, \cdots, m$. Here $e_{j}$ is an $(m-2) \times 1$ vector with the $(j-1)$-th component as 1 and all other components as $0, \phi_{j}(u, z)=\phi\left\{\tau_{j}-H(u)-\theta z\right\}$, and $\phi$ is the probability density function of the standard normal random variable. Define 


$$
\begin{aligned}
& T_{0}(u)=\sum_{z \in Q} \sum_{j=1}^{m} d_{j}(u, z)^{2} \psi_{j}(u, z) f(u, z) / D_{j}(u, z), \\
& T_{1}(u)=\sum_{z \in Q} \sum_{j=1}^{m} d_{j}(u, z)^{2} \psi_{j}(u, z) z^{T} f(u, z) / D_{j}(u, z), \\
& T_{2}(u)=\sum_{z \in Q} \sum_{j=1}^{m} d_{j}(u, z)^{2} \psi_{j}(u, z) \psi_{j}(u, z)^{T} f(u, z) / D_{j}(u, z), \\
& \Gamma_{j}=\int_{0}^{1} \cdots \int_{0}^{1} T_{j}(u) d u, \text { for } j=0,1,2,
\end{aligned}
$$

where $D_{j}(u, z)$ is $d_{j}(u, z)$ with $\phi$ replaced by $\Phi$. Similarly define $\xi_{0}, \xi_{1}$, and $\xi_{2}$ as $\kappa_{0}$, $\kappa_{1}$, and $\kappa_{2}$ with $K^{\#}(u)$ replaced by $K^{\#}(u)^{2}$, define $S_{0}(u), S_{1}(u)$, and $S_{2}(u)$ as $T_{0}(u)$, $T_{1}(u)$, and $T_{2}(u)$ with $\psi_{j}(u, z), \psi_{j}(u, z) z^{T}$, and $\psi_{j}(u, z) \psi_{j}(u, z)^{T}$ replaced by $1, z$, and $\mathrm{Z} \mathrm{z}^{T}$, respectively in each case, and define $\Sigma_{j}=\int_{0}^{1} \cdots \int_{0}^{1} S_{j}(u) d u$, for $j=0,1,2$.

Finally, define quantities related to asymptotic biases and variances of the estimators $\hat{\theta}, \hat{\tau}$, and $\hat{H}\left(x_{0}\right)$ :

$$
\begin{aligned}
& b_{\theta}=G^{-1}\left(\Gamma_{1}^{T} \Gamma_{2}^{-1} \lambda_{0}-\lambda_{1}\right), \quad V_{\theta}=G^{-1}, \\
& b_{\tau}=\Gamma_{2}^{-1}\left(\Gamma_{1} b_{\theta}+\lambda_{0}\right), \quad V_{\tau}=\Gamma_{2}^{-1} \Gamma_{1} G^{-1} \Gamma_{1}^{T} \Gamma_{2}^{-1}+\Gamma_{2}^{-1}, \\
& b_{H}\left(x_{0}\right)=\frac{\left(\kappa_{2}^{*}\right)^{1-d} \operatorname{det}\left(\kappa_{2}^{*} \kappa_{2}-\kappa_{3}^{*} \kappa_{1}^{T}\right)}{2\left(\kappa_{0}\right)^{1-d} \operatorname{det}\left(\kappa_{0} \kappa_{2}-\kappa_{1} \kappa_{1}^{T}\right)}, \quad V_{H}\left(x_{0}\right)=\frac{\xi_{0}-2 \kappa_{1}^{T} \kappa_{2}^{-1} \xi_{1}+\kappa_{1}^{T} \kappa_{2}^{-1} \xi_{2} \kappa_{2}^{-1} \kappa_{1}}{S_{0}\left(x_{0}\right)\left(\kappa_{0}-\kappa_{1}^{T} \kappa_{2}^{-1} \kappa_{1}\right)^{2}},
\end{aligned}
$$

where

$$
\begin{aligned}
& G=\Sigma_{2}-\Gamma_{1}^{T} \Gamma_{2}^{-1} \Gamma_{1}, \\
& \lambda_{0}=(1 / 2)\left\{\int_{-1}^{1} t^{2} K(t) d t\right\} \int_{0}^{1} \cdots \int_{0}^{1}\left\{\sum_{j=1}^{d} H_{j, j}(u)\right\} T_{0}(u) d u, \\
& \lambda_{1}=(1 / 2)\left\{\int_{-1}^{1} t^{2} K(t) d t\right\} \int_{0}^{1} \cdots \int_{0}^{1}\left\{\sum_{j=1}^{d} H_{j, j}(u)\right\} S_{1}(u) d u .
\end{aligned}
$$

Specifically, if $x_{0}$ is in the interior region $[g, 1-g]^{d}$ of the support $[0,1]^{d}$ of the marginal probability density function of the d-dimensional continuous explanatory variable $X$, then

$$
b_{H}\left(x_{0}\right)=(1 / 2)\left\{\int_{-1}^{1} t^{2} K(t) d t\right\}\left\{\sum_{j=1}^{d} H_{j, j}\left(x_{0}\right)\right\}, \quad V_{H}\left(x_{0}\right)=S_{0}\left(x_{0}\right)^{-1}\left\{\int_{-1}^{1} K(t)^{2} d t\right\}^{d} .
$$

The following Theorem 1 states the asymptotic bias and variance for each of $\hat{\theta}, \hat{\tau}$, and $\hat{H}\left(x_{0}\right)$. Its proof will be given in Appendix B. 
Theorem 1. Assume the model (7), and let conditions (C1)-(C4) be satisfied. The asymptotic biases and variances of $\hat{\theta}$ and $\hat{\tau}$ can be expressed as

$$
\begin{array}{ll}
\operatorname{Bias}(\hat{\theta})=b^{2} b_{\theta}\{1+o(1)\}, & \operatorname{Var}(\hat{\theta})=n^{-1} V_{\theta}\{1+o(1)\}, \\
\operatorname{Bias}(\hat{\tau})=b^{2} b_{\tau}\{1+o(1)\}, & \operatorname{Var}(\hat{\tau})=n^{-1} V_{\tau}\{1+o(1)\} .
\end{array}
$$

Also, those of $\hat{H}\left(x_{0}\right)$ can be expressed as

$$
\operatorname{Bias}\left\{\hat{H}\left(x_{0}\right)\right\}=g^{2} b_{H}\left(x_{0}\right)\{1+o(1)\}, \quad \operatorname{Var}\left\{\hat{H}\left(x_{0}\right)\right\}=n^{-1} g^{-d} V_{H}\left(x_{0}\right)\{1+o(1)\},
$$

for each $x_{0} \in[0,1]^{d}$.

Remark 1. By Theorem 2.1 of Ruppert and Wand (1994) and our (A3) in Theorem 1, the optimal $K$ satisfying the conditions in (C3) for constructing $\hat{H}\left(x_{0}\right)$, for each $x_{0} \in[0,1]^{d}$, is the Epanechnikov kernel defined as $K(t)=(3 / 4)\left(1-t^{2}\right) I(|t| \leq 1)$, in the sense of having smaller asymptotic mean integrated square error. On the other hand, by (A3), the optimal choice of the value of $g$, in terms of having smaller asymptotic mean integrated square error of $\hat{H}\left(x_{0}\right)$, is

$$
g^{*}=c_{0}^{1 /(d+4)} n^{-1 /(d+4)},
$$

where $c_{0}=\left\{d \int_{0}^{1} \cdots \int_{0}^{1} V_{H}(u) d u\right\}\left\{4 \int_{0}^{1} \cdots \int_{0}^{1} b_{H}(u)^{2} d u\right\}^{-1}$. However, the optimal value $g^{*}$ is not available in practice since it depends on the unknown factors $H(x), \theta, \tau$, and $f(x, z)$. Similarly, by (A1)-(A3) and (C4), the optimal value $b^{*}$ of $b$ for constructing $\tilde{H}\left(x_{0}\right)$, in terms of having smaller asymptotic mean square error of $\hat{\theta}$ and $\hat{\tau}$, satisfies the condition $n^{-1 /(d+4)}>>b>n^{-1 /(d+2)}$. Thus we conclude that the value of $g^{*}$ is of larger order than that of $b^{*}$, and that the asymptotic mean integrated square error of $\hat{H}\left(x_{0}\right)$ using $g^{*}$ is of smaller order in magnitude than that of $\tilde{H}\left(x_{0}\right)$ using $b^{*}$. The specific formulation of the asymptotic bias and variance of $\tilde{H}\left(x_{0}\right)$ will be given in (A10) in Appendix B.

\section{Appendix B. Sketches of the Proofs}

In Appendix B, sketches of the proof for Theorem 1 will be given. The following notation will be used throughout Appendix B. Set $\omega=(\eta, \theta, \tau), \quad \tilde{\omega}=(\tilde{\eta}, \tilde{\theta}, \tilde{\tau}), \quad \zeta=(\theta, \tau)$, and $\hat{\zeta}=(\hat{\theta}, \hat{\tau})$. Let $\ell_{\mathrm{j}}^{*(1)}$ and $\ell_{\mathrm{j}}^{*(2)}$ be the gradient vector and the Hessian matrix of the loglikelihood function $\ell_{\mathrm{j}}^{*}$ given in Sections 2.3-2.4, respectively, for each $j=0,1,2$. Also, let 
$H^{(2)}$ be the Hessian matrix of $H$. Let $f_{X}$ be the marginal probability density function of the ddimensional continuous explanatory variable $X$. Define $P_{0}$ as the event that the number of $x_{i}$ falling into the region $\prod_{j=1}^{d}\left[x_{0 j}-b / 2, x_{0 j}+b / 2\right]$ is less than $\rho_{0} n \int_{x_{01}-b / 2}^{x_{01}+b / 2} \cdots \int_{x_{0 d}-b / 2}^{x_{0 d}+b / 2} f_{X}(u) d u$, where $\rho_{0}$ is a positive constant satisfying $\rho_{0} \leq 1 / 4$, and $Q_{0}$ the event that the number of $x_{i}$ falling into the region $\prod_{j=1}^{d}\left[x_{0 j}-b, x_{0 j}+b\right]$ is greater than $\varphi_{0} n \int_{x_{01}-b}^{x_{01}+b} \cdots \int_{x_{0 d}-b}^{x_{00}+b} f_{X}(u) d u$, where $\varphi_{0}$ is a positive constant $\varphi_{0} \geq \exp (1)$.

Proof of the asymptotic bias and variance of $\tilde{H}\left(x_{0}\right)$. By the first order Taylor theorem, we have

$$
0=\ell_{0}^{*(1)}\left(\tilde{\omega} ; x_{0}\right)=\ell_{0}^{*(1)}\left(\omega ; x_{0}\right)+\ell_{0}^{*(2)}\left(\omega^{*} ; x_{0}\right)(\tilde{\omega}-\omega),
$$

for each $x_{0} \in[0,1]^{d}$, where $\omega^{*}$ lies in the line segment connecting $\omega$ and $\tilde{\omega}$.

Using (C1)-(C4), the large deviation theorem in Section 10.3.1 of Serfling (1980), and approximations to the standard errors of functions of random variables in Section 10.5 of Stuart and Ord (1987), a straightforward calculation leads to the following asymptotic results: as $n \rightarrow \infty$,

$$
\begin{aligned}
& \operatorname{Pr}\left(P_{0} \cup Q_{0}\right)=O\{\exp (-n b)\}, \\
& \mathrm{E}\left\{\ell_{0}^{*(1)}\left(\omega ; x_{0}\right)\right\}=(1 / 2) n b^{2} A_{0}\{1+o(1)\}, \\
& \mathrm{E}\left\{\ell_{0}^{*(2)}\left(\omega ; x_{0}\right)\right\}=(-1) n B_{0}\{1+o(1)\}, \\
& \operatorname{Var}\left\{\ell_{0}^{*}(1)\left(\omega ; x_{0}\right)\right\}=n b^{-d} C_{0}\{1+o(1)\},
\end{aligned}
$$

for each $\omega$, where the little-o terms in (A6)-(A8) all tend to zero uniformly in $x_{0}$,

$$
A_{0}=\left[\begin{array}{c}
\kappa_{2}^{*} S_{0} \\
b \kappa_{3}^{*} S_{0} \\
\kappa_{2}^{*} S_{1} \\
(-1) \kappa_{2}^{*} T_{0}
\end{array}\right], \quad B_{0}=\left[\begin{array}{cccc}
\kappa_{0} S_{0}, & b S_{0} \kappa_{1}^{T}, & \kappa_{0} S_{1}^{T}, & (-1) \kappa_{0} T_{0}^{T} \\
b S_{0} \kappa_{1}, & b^{2} S_{0} \kappa_{2}, & b \kappa_{1} S_{1}^{T}, & (-b) \kappa_{1} T_{0}^{T} \\
\kappa_{0} S_{1}, & b S_{1} \kappa_{1}^{T}, & \kappa_{0} S_{2}, & (-1) \kappa_{0} T_{1}^{T} \\
(-1) \kappa_{0} T_{0}, & (-b) T_{0} \kappa_{1}^{T}, & (-1) \kappa_{0} T_{1}, & \kappa_{0} T_{2}
\end{array}\right],
$$

and $C_{0}$ is $B_{0}$ with each element $\kappa_{j}$ replaced by $\xi_{j}$, for $j \geq 0$.

Using the results of (A4)-(A8) and comparing the magnitudes of $\ell_{0}^{*(1)}\left(\omega ; x_{0}\right)=O_{p}\left(n b^{2}+n^{1 / 2} b^{-d / 2}\right)$ and $\ell_{0}^{*(2)}\left(\omega^{*} ; x_{0}\right)=O_{p}(n)$ in (A4), we have

$$
\tilde{\omega}-\omega=O_{p}\left(b^{2}+n^{-1 / 2} b^{-d / 2}\right) .
$$


Using (A4)-(A9) and approximations to the standard errors of functions of random variables and Theorem A.3 of Anderson (2003), the asymptotic bias and variance of the estimator $\tilde{H}\left(x_{0}\right)$ can be expressed as

$$
\operatorname{Bias}\left\{\tilde{H}\left(x_{0}\right)\right\}=b^{2} b_{H}\left(x_{0}\right)\{1+o(1)\}, \quad \operatorname{Var}\left\{\tilde{H}\left(x_{0}\right)\right\}=n^{-1} b^{-d} V_{0}\left(x_{0}\right)\{1+o(1)\},
$$

for each $x_{0} \in[0,1]^{d}$. Here the little-o terms in (A10) all tend to zero uniformly in $x_{0}$, and $V_{0}\left(x_{0}\right)$ is the $(1,1)$-th component of the matrix $B_{0}^{-1} C_{0} B_{0}^{-1}$. The proof of the asymptotic bias and variance of $\tilde{H}\left(x_{0}\right)$ is complete.

Proof of (A1) and (A2). By the first order Taylor theorem, we have

$$
0=\ell_{1}^{*(1)}(\hat{\zeta})=\ell_{1}^{*(1)}(\zeta)+\ell_{1}^{*(2)}\left(\zeta^{*}\right)(\hat{\zeta}-\zeta),
$$

where $\zeta^{*}$ lies in the line segment connecting $\zeta$ and $\hat{\zeta}$. Using (A10), (C1)-(C4), and approximations to the standard errors of functions of random variables, a straightforward calculation leads to the following asymptotic results: as $n \rightarrow \infty$,

$$
\begin{aligned}
& \mathrm{E}\left\{\ell_{1}^{*(1)}(\zeta)\right\}=n b^{2} A_{1}\{1+o(1)\}, \\
& \mathrm{E}\left\{\ell_{1}^{*(2)}(\zeta)\right\}=(-1) n C_{1}\{1+o(1)\}, \\
& \operatorname{Var}\left\{\ell_{1}^{*}(1)(\zeta)\right\}=n C_{1}\{1+o(1)\},
\end{aligned}
$$

for each $\zeta$, where

$$
A_{1}=\left[\begin{array}{c}
(-1) \lambda_{1} \\
\lambda_{0}
\end{array}\right], \quad C_{1}=\left[\begin{array}{cc}
\Sigma_{2}, & (-1) \Gamma_{1}^{T} \\
(-1) \Gamma_{1}, & \Gamma_{2}
\end{array}\right] .
$$

Following the same argument of (A4)-(A9) and using (A11)-(A14), we have

$$
\hat{\zeta}-\zeta=O_{p}\left(b^{2}+n^{-1 / 2}\right) .
$$

Combining this result with (A12)-(A14) and (C1)-(C4), and using approximations to the standard errors of functions of random variables and Theorem A.3 of Anderson (2003), the results of (A1) and (A2) follow.

Proof of (A3). Using (C1)-(C4), (A1)-(A2), and approximations to the standard errors of functions of random variables, a straightforward calculation leads to the following asymptotic results: as $n \rightarrow \infty$,

$$
\begin{aligned}
& \mathrm{E}\left\{\ell_{2}^{*(1)}\left(\eta ; x_{0}\right)\right\}=(1 / 2) n g^{2} A_{2}\{1+o(1)\}, \\
& \mathrm{E}\left\{\ell_{2}^{*(2)}\left(\eta ; x_{0}\right)\right\}=(-1) n B_{2}\{1+o(1)\}, \\
& \operatorname{Var}\left\{\ell_{2}^{*(2)}\left(\eta ; x_{0}\right)\right\}=n g^{-d} C_{2}\{1+o(1)\},
\end{aligned}
$$


for each $\eta$, where the little-o terms in (A16)-(A18) all tend to zero uniformly in $x_{0}$,

$$
A_{2}=\left[\begin{array}{c}
\kappa_{2}^{*} S_{0} \\
g \kappa_{3}^{*} S_{0}
\end{array}\right], \quad B_{2}=\left[\begin{array}{cc}
\kappa_{0} S_{0}, & g S_{0} \kappa_{1}^{T} \\
g S_{0} \kappa_{1}, & g^{2} S_{0} \kappa_{2}
\end{array}\right],
$$

and $C_{2}$ is $B_{2}$ with each element $\kappa_{j}$ replaced by $\xi_{j}$, for $j \geq 0$. Following the same argument of (A4)-(A9) and using (A16)-(A18), we have $\hat{\eta}-\eta=O_{p}\left(g^{2}+n^{-1 / 2} g^{-d / 2}\right)$. Combining this result with (A16)-(A18), and using approximations to the standard errors of functions of random variables and Theorem A.3 of Anderson (2003), the result of (A3) follows.

\section{References}

Anderson, T.W., 2003. An Introduction to Multivariate Statistical Analysis. Wiley, New Jersey.

Allison, P.D., 2001. Missing data. SAGE Publications, London.

Altman, E.I., 1968. Financial ratios, discriminant analysis, and the prediction of corporate bankruptcy. Journal of Finance, 23, 589-609.

Altman, E.I., Avery, R.B., Eisenbeis, R.A., Sinkey, J.F., 1981. Application of Classification Techniques in Business, Banking and Finance. JAI Press, Connecticut.

Altman, E.I., Katz, S., 1976. Statistical bond rating classification using financial and accounting data. In: Schiff, M., Sorter, G. (Eds.), Proceedings of the Conference on Topical Research in Accounting. New York University Press, New York, pp. 205-239.

Begley, J., Ming, J., Watts, S., 1996. Bankruptcy classification errors in the 1980s: An empirical analysis of Altman's and Ohlson's models. Review of Accounting Studies, 1, 267-284.

Bharath, S.T., Shumway, T., 2008. Forecasting default with the Merton distance to default model. Review of Financial Studies, 21, 1339-1369.

Blume, M.E., Lim, F., MacKinlay, A.C., 1998. The declining credit quality of U.S. corporate debt: Myth or reality? Journal of Finance, 53, 1389-1414.

Borooah, V.K., 2002. Logit and Probit. SAGE Publications, London.

Chava, S., Jarrow, R.A., 2004. Bankruptcy prediction with industry effects. Review of Finance, 8, 537-569.

Chava, S., Stefanescu, C., Turnbull, S., 2008. Modeling the loss distribution. Available at http://faculty.london.edu/cstefanescu/research.html.

Duffie, D., Eckner, A., Horel, G., Saita, L., 2006. Frailty Correlated Default. Available at http://www.stanford.edu/ duffie/working.htm.

Ederington, L.H., 1985. Classification models and bond ratings. The Financial Review, 20, 237262. 
Eubank, R.L., 1988. Spline Smoothing and Nonparametric Regression. Marcel Dekker, New York.

Fan, J., Gijbels, I., 1996. Local Polynomial Modeling and Its Application - Theory and Methodologies. Chapman and Hall, New York.

Fan, J., Heckman, N.E., Wand, M.P., 1995. Local polynomial kernel regression for generalized linear models and quasi-likelihood functions. Journal of the American Statistical Association, 90, 141-150.

Galil, K., 2003. The quality of corporate credit rating: An empirical investigation. Available at http://ssrn.com/abstract=406681.

Gentry, J.A., Whitford, D.T., Newbold, P., 1988. Predicting industrial bond ratings with a probit model and fund flow components. The Financial Review, 23, 269-286.

Greene, W.H., 2002. LIMDEP Version 8.0 Econometric Modeling Guide. Econometric Software Inc., New York.

Güttler, A., Wahrenburg, M., 2007. The adjustment of credit ratings in advance of defaults. Journal of Banking and Finance, 31, 751-767.

Härdle, W., 1990. Applied Nonparametric Regression. Cambridge University Press, Cambridge.

Härdle, W., 1991. Smoothing Techniques: With Implementation in S. Springer, Berlin.

Härdle, W., Moro, R.A., Schäfer, D., 2006. Graphical data representation in bankruptcy analysis. In: Chen, C.H., Härdle, W., Unwin, A. (Eds.), Handbook for Data Visualization. Springer, Berlin.

Härdle, W., Hart, J., Marron, J.S., Tsybakov, A.B., 1992. Bandwidth selection for average derivative estimation. Journal of the American Statistical Association, 87, 218-226.

Horrigan, J.O., 1966. The determination of long-term credit standing with financial ratios. Journal of Accounting Research, 4, 44-62.

Huang, Z., Chen, H., Hsu, C.J., Chen, W.H., Wu, S., 2004. Credit rating analysis with support vector machines and neural networks: a market comparative study. Decision Support Systems, 37, 543-558.

Hwang, R.C., Cheng, K.F., Lee, J.C., 2007. A semiparametric method for predicting bankruptcy. Journal of Forecasting, 26, 317-342.

Hwang, R.C., Cheng, K.F., Lee, C.F., 2008. On multiple-class prediction of issuer credit ratings. To appear in Applied Stochastic Models in Business and Industry. Available at: http://www3.interscience.wiley.com/cgi-bin/fulltext/121392086/PDFSTART.

Johnson, R.A., Wichern, D.W., 2002. Applied Multivariate Statistical Analysis. Prentice Hall, New York.

Jones, M.C., Marron, J.S., Sheather, S.J., 1996. A brief survey of bandwidth selection for density estimation. Journal of the American Statistical Association, 91, 401-407. 
Kaplan, R.S., Urwitz, G., 1979. Statistical models of bond ratings: A methodological inquiry. Journal of Business, 52, 231-261.

Lipsitz, S.R., Kim, K., Zhao, L., 1994. Analysis of repeated categorical data using generalized estimating equations. Statistics in Medicine, 13, 1149-1163.

Little, R.J.A., Rubin, D.B., 2002. Statistical Analysis with Missing Data. Wiley, New York.

Marron, J.S., Wand, M.P., 1992. Exact mean integrated square error. Annals of Statistics, 20, 712-736.

McCullagh, P., 1980. Regression models for ordinal data. Journal of the Royal Statistical Society, Series B, 42, 109-142.

Müller, H.G., 1988. Nonparametric Regression Analysis of Longitudinal Data. Springer, Berlin.

Müller, G., Czado, C., 2005. An autoregressive ordered probit model with application to high frequency financial data. Journal of Computational and Graphical Statistics, 14, 320-338.

Ohlson, J., 1980. Financial ratios and the probabilistic prediction of bankruptcy. Journal of Accounting Research, 18, 109-131.

Pettit, J., Fitt, C., Orlov, S., Kalsekar, A., 2004. The new world of credit ratings. Available at http://ssrn.com/abstract=593522.

Pinches, G.E., Mingo, K.A., 1973. A multivariate analysis of industrial bond ratings. Journal of Finance, 28, 1-18.

Pinches, G.E., Mingo, K.A., 1975. A note on the role of subordination in determining industrial bond ratings. Journal of Finance, 30, 201-206.

Pogue, T.F., Soldofsky, R.M., 1969. What's in a bond rating? Journal of Financial and Quantitative Analysis, 4, 201-228.

Poon, W.P.H., 2003. Are unsolicited credit ratings biased downward? Journal of Banking and Finance, 27, 593-614.

Ruppert, D., Wand, M.P., 1994. Multivariate locally weighted least squares regression. Annals of Statistics, 22, 1346-1370.

Scott, D.W., 1992. Multivariate Density Estimation: Theory, Practice, and Visualization. Wiley, New York.

Serfling, R.J., 1980. Approximation Theorems of Mathematical Statistics. Wiley, New York.

Shumway, T., 2001. Forecasting bankruptcy more accurately: a simple hazard model. Journal of Business, 74, 101-124.

Simonoff, J.S., 1996. Smoothing Methods in Statistics. Springer, New York.

Standard \& Poor's, 2004. Standard \& Poor’s Research Insight North America Data Guide. McGraw-Hill, New York.

Staniswallis, J.G., 1989. The kernel estimate of a regression function in likelihood-based models. Journal of the American Statistical Association, 84, 276-283. 
Stuart, A., Ord, J.K., 1987. Kendall's Advanced Theory of Statistics, Volume 1. Oxford University Press, New York.

Tibshirani, R., Hastie, T., 1987. Local likelihood estimation. Journal of the American Statistical Association, 82, 559-568.

Wand, M.P., Jones, M.C., 1995. Kernel Smoothing. Chapman and Hall, London.

West, R.R., 1970. An alternative approach to predicting corporate bond ratings. Journal of Accounting Research, 8, 118-125.

Wijayatunga, P., Mase, S., Nakamura, M., 2006. Appraisal of companies with Bayesian Networks. International Journal of Business Intelligence and Data Mining, 1, 329-346. 

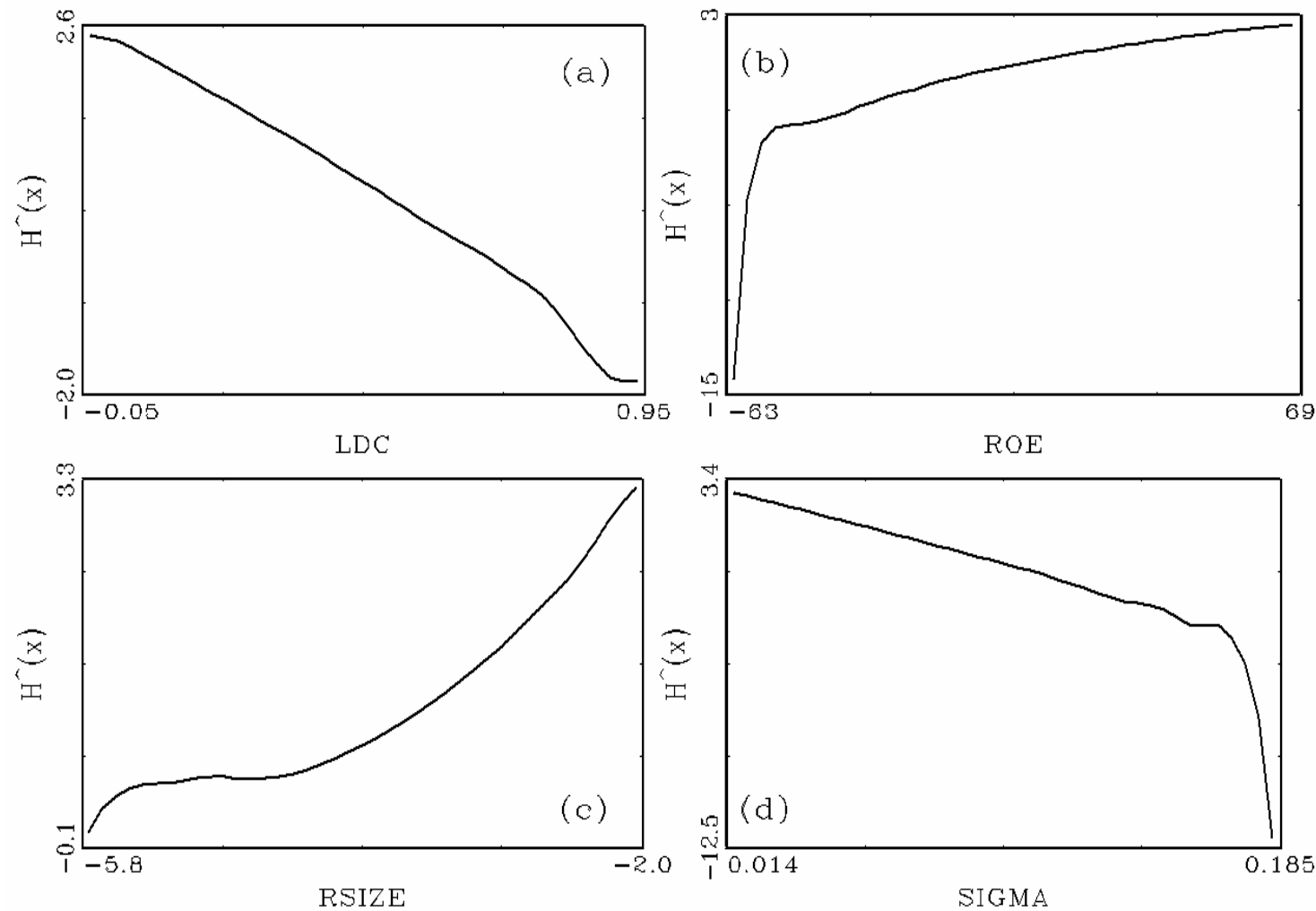

Figure 1: Plots of marginal relations between the function $\hat{H}(x)$ and the four selected continuous predictors LDC, ROE, RSIZE, and SIGMA. Panels (a)-(d) show the plots of $\left\{x_{j}, \hat{H}(x)\right\}$ resulted from OSPM using $\left\{\hat{b}(1), \hat{g}(1), \hat{p}_{\text {OSPM }}(1)\right\}$. Here $x_{j}$ in the plot of $\left\{x_{j}, \hat{H}(x)\right\}$ stands for the value of the $j$-th selected continuous predictor, and $x$ has the $j$-th component as $x_{j}$, but all other components are fixed at their sample median levels. From panels (a)-(d), the slope of each curve agrees with the expected direction of the corresponding variable effect. But these plots show clearly that there is a nonlinear relationship between $x_{j}$ and $\hat{H}(x)$ for each of ROE, RSIZE, and SIGMA, except LDC. 

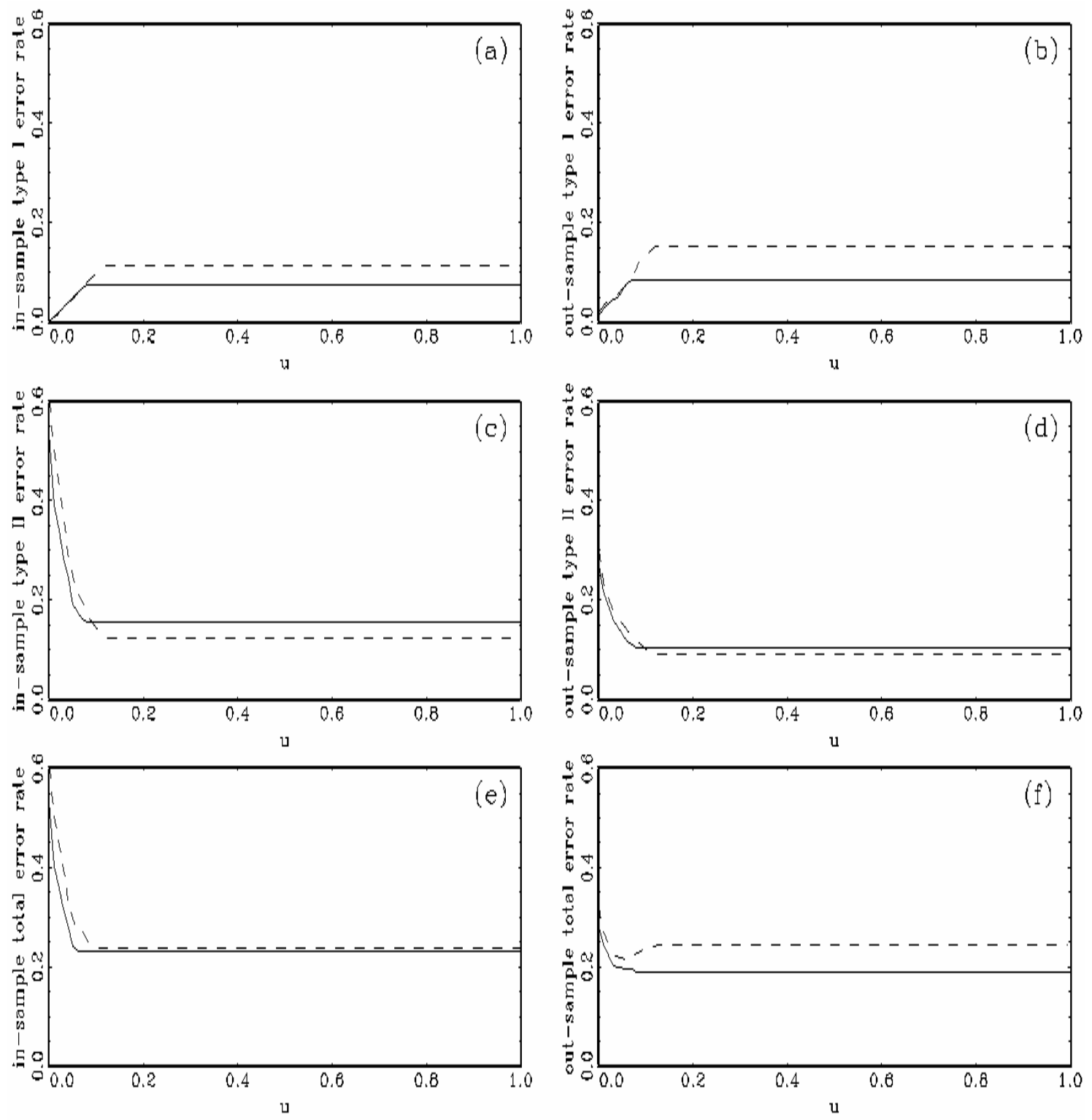

Figure 2: The performance of the two prediction rules based on OLPM (dashed curve) and OSPM (solid curve) using the five selected variables LDC, ROE, RSIZE, SIGMA, and industry effects. Panels (a), (c), and (e) show respectively the in-sample type I, type II, and total error rates obtained from the 413 estimation companies by the two prediction rules. Panels (b), (d), and (f) show respectively the out-of-sample type I, type II, and total error rates derived from the 366 holdout companies by the two prediction rules. 
Table 1. The frequency distributions of the sampled companies collected from the COMPUSTAT and CRSP databases with complete values of the twenty-four potential predictors for studying S\&P's LTRs in April 2007. Panels A, B, and C present the frequency distributions of the sampled companies according to different S\&P’s LTR categories, and SIC codes.

\begin{tabular}{lll}
\hline & Estimation companies & Holdout companies \\
\hline
\end{tabular}

Panel A: S\&P's LTR

$\begin{array}{lcc}\text { AAA } & 4 & 2 \\ \text { AA } & 18 & 3 \\ \text { A } & 88 & 32 \\ \text { BBB } & 156 & 78 \\ \text { BB } & 89 & 133 \\ \text { B } & 57 & 108 \\ \text { CCC } & 0 & 9 \\ \text { CC } & 1 & 0 \\ \text { C } & 0 & 0 \\ \text { D } & 0 & 1 \\ \text { Total firms } & 413 & 366\end{array}$

Panel B: S\&P's LTR Category

$\begin{array}{lcc}\{\text { Below BBB }\} & 147 & 251 \\ \{\text { BBB } & 156 & 78 \\ \{\text { AAA, AA, A }\} & 110 & 37 \\ \text { Total firms } & 413 & 366\end{array}$

Panel C: SIC code

1000 1999 $37 \quad 50$

2000 2999 $112 \quad 67$

3000 3999 $\quad 93 \quad 85$

4000 4999 $114 \quad 96$

$5000 \sim 5999 \quad 20 \quad 18$

$7000 \sim 7999 \quad 26 \quad 31$

8000 8999 $11 \quad 19$

Total firms $413 \quad 366$

Note: The financial service companies with SIC code 6000-6999 were excluded from the study, since they are subject to regulations and adopt different accounting conventions. There were only three companies having SIC code less than 1000 and two companies carrying SIC code greater than 9000. These five companies were also excluded from study. If those industries with few companies are included in the prediction model, then the resulting number of industry indicator variables is increased, thus the estimates of parameters in the corresponding model might become less precise. 
Table 2. The definitions of the twenty-four potential predictors for studying important predictors of S\&P's LTRs in April 2007. Panels A, B, and C present the definitions of market-driven variables, accounting variables, and industry indicator variables, respectively.

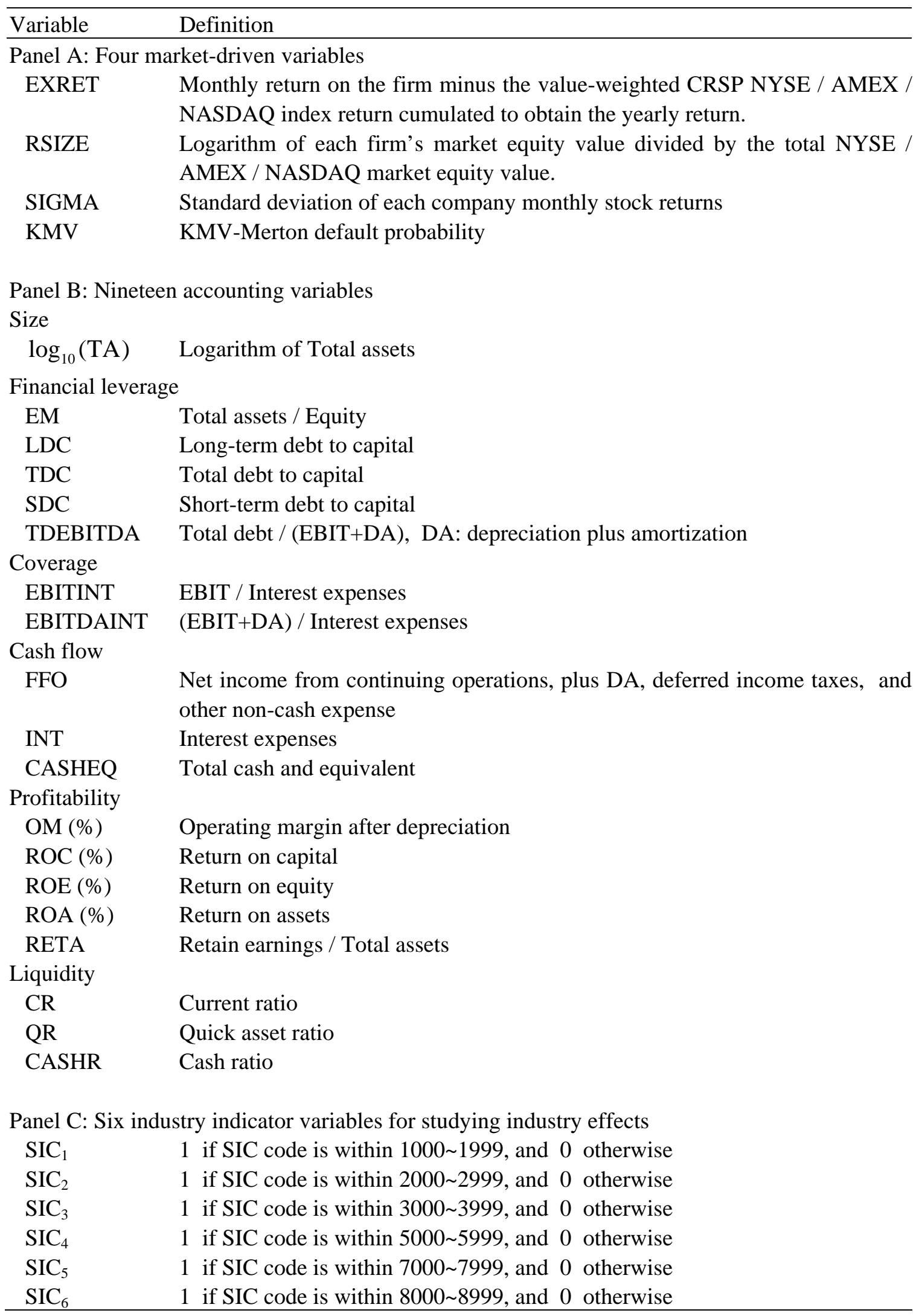

Note: The SIC code 4000 4999 was used as the reference level in studying the industry effects. 
Table 3. Summary statistics and F-tests of the estimation sample. The p-values refer to the Ftests of equality of the means among the three S\&P's LTR categories based on the estimation sample. Panels A, B, and C present the results for the three S\&P's LTR categories $\{$ Below BBB\}, $\{\mathrm{BBB}\}$, and $\{\mathrm{AAA}, \mathrm{AA}, \mathrm{A}\}$, respectively.

\begin{tabular}{|c|c|c|c|c|c|c|}
\hline Variable & Mean & Median & $\begin{array}{l}\text { Standard } \\
\text { deviation }\end{array}$ & Minimum & Maximum & $p$-value \\
\hline \multicolumn{7}{|c|}{ Panel A: \{Below BBB $\}$} \\
\hline EXRET & 0.115 & 0.070 & 0.292 & -0.422 & 1.054 & $0.007 * *$ \\
\hline RSIZE & -4.076 & -4.036 & 0.564 & -6.548 & -2.898 & $0.000^{* *}$ \\
\hline SIGMA & 0.099 & 0.093 & 0.038 & 0.044 & 0.251 & $0.000^{* *}$ \\
\hline KMV & 0.029 & 0.000 & 0.105 & 0.000 & 0.966 & $0.007 * *$ \\
\hline $\log _{10}$ (TA) & 3.425 & 3.420 & 0.480 & 2.350 & 4.478 & $0.000^{* *}$ \\
\hline EM & 4.749 & 3.058 & 13.916 & -60.917 & 139.570 & 0.096 \\
\hline LDC & 0.486 & 0.468 & 0.208 & 0.002 & 1.013 & $0.000 * *$ \\
\hline TDC & 0.530 & 0.513 & 0.205 & 0.028 & 1.023 & $0.000 * *$ \\
\hline SDC & 0.044 & 0.021 & 0.075 & 0.000 & 0.462 & $0.012 *$ \\
\hline TDEBITDA & 3.633 & 3.310 & 9.613 & -80.917 & 69.617 & $0.026^{*}$ \\
\hline EBITINT & 5.775 & 2.637 & 15.846 & -25.763 & 164.612 & $0.000^{* *}$ \\
\hline EBITDAINT & 9.724 & 4.063 & 33.768 & -18.618 & 394.721 & $0.000^{* *}$ \\
\hline FFO & 352.271 & 181.167 & 496.760 & -280.233 & 3065.000 & $0.000^{* *}$ \\
\hline INT & 149.658 & 63.678 & 244.430 & 1.770 & 1915.667 & $0.000^{* *}$ \\
\hline CASHEQ & 351.692 & 145.858 & 566.090 & 0.179 & 3641.667 & $0.000^{* *}$ \\
\hline $\mathrm{OM}$ & 11.453 & 8.450 & 14.927 & -83.099 & 49.102 & $0.000^{* *}$ \\
\hline ROC & 3.313 & 3.464 & 9.609 & -61.724 & 34.882 & $0.000^{* *}$ \\
\hline ROE & 3.804 & 6.582 & 40.686 & -211.23 & 242.545 & $0.000 * *$ \\
\hline ROA & 2.303 & 2.491 & 5.331 & -24.334 & 19.009 & $0.000^{* *}$ \\
\hline RETA & 0.016 & 0.070 & 0.373 & -2.016 & 0.763 & $0.000^{* *}$ \\
\hline CR & 1.741 & 1.524 & 1.098 & 0.472 & 11.247 & $0.026^{*}$ \\
\hline QR & 1.165 & 0.980 & 0.953 & 0.271 & 9.855 & 0.090 \\
\hline CASHR & 0.559 & 0.344 & 0.901 & 0.005 & 9.154 & 0.055 \\
\hline
\end{tabular}

Panel B: $\{B B B\}$

$\begin{array}{lccccc}\text { EXRET } & 0.067 & 0.050 & 0.148 & -0.336 & 0.630 \\ \text { RSIZE } & -3.598 & -3.609 & 0.519 & -5.410 & -2.325 \\ \text { SIGMA } & 0.061 & 0.059 & 0.019 & 0.026 & 0.114 \\ \text { KMV } & 0.003 & 0.000 & 0.019 & 0.000 & 0.219\end{array}$

(continued on next page) 
Table 3. (continued)

\begin{tabular}{lcccccc}
\hline Variable & Mean & Median & $\begin{array}{c}\text { Standard } \\
\text { deviation }\end{array}$ & Minimum & Maximum & $p$-value \\
\hline $\log _{10}(\mathrm{TA})$ & 3.822 & 3.785 & 0.456 & 2.947 & 5.100 & 17.591 \\
EM & 3.030 & 2.643 & 1.794 & 1.433 & 0.822 \\
LDC & 0.350 & 0.345 & 0.139 & 0.002 & 0.873 \\
TDC & 0.406 & 0.395 & 0.150 & 0.035 & 0.225 \\
SDC & 0.056 & 0.045 & 0.048 & 0.000 & 17.142 \\
TDEBITDA & 2.382 & 1.986 & 1.698 & 0.097 & 171.302 \\
EBITINT & 9.607 & 5.781 & 17.731 & 0.713 & 248.009 \\
EBITDAINT & 13.342 & 8.500 & 24.626 & 1.602 & 16427.302 \\
FFO & 1046.719 & 522.681 & 1619.155 & 17.967 & 1.345 & 2974.245 \\
INT & 209.605 & 96.369 & 335.349 & 1159 & \\
CASHEQ & 651.585 & 285.864 & 1151.857 & 4.074 & 10287.194 \\
OM & 14.846 & 12.764 & 8.828 & 1.279 & 52.942 \\
ROC & 8.299 & 7.638 & 5.847 & -17.410 & 38.158 \\
ROE & 14.591 & 13.219 & 11.126 & -22.747 & 97.052 \\
ROA & 5.217 & 4.762 & 3.357 & -12.769 & 14.676 \\
RETA & 0.212 & 0.219 & 0.189 & -0.747 & 0.758 \\
CR & 1.492 & 1.395 & 0.715 & 0.279 & 4.839 \\
QR & 0.982 & 0.853 & 0.560 & 0.159 & 4.035 \\
CASHR & 0.376 & 0.220 & 0.456 & 0.009 & 3.487
\end{tabular}

Panel C: $\{$ AAA, AA, A

$\begin{array}{lccccc}\text { EXRET } & 0.032 & 0.012 & 0.135 & -0.241 & 0.516 \\ \text { RSIZE } & -3.104 & -3.061 & 0.611 & -4.907 & -1.678 \\ \text { SIGMA } & 0.053 & 0.052 & 0.017 & 0.025 & 0.106 \\ \text { KMV } & 0.010 & 0.000 & 0.070 & 0.000 & 0.553 \\ \log _{10}(\mathrm{TA}) & 4.131 & 4.121 & 0.563 & 2.522 & 5.317 \\ \text { EM } & 2.701 & 2.314 & 1.303 & 1.245 & 9.300 \\ \text { LDC } & 0.271 & 0.245 & 0.153 & 0.001 & 0.707 \\ \text { TDC } & 0.339 & 0.333 & 0.174 & 0.004 & 0.771 \\ \text { SDC } & 0.067 & 0.058 & 0.057 & 0.000 & 0.286 \\ \text { TDEBITDA } & 1.686 & 1.391 & 1.415 & 0.016 & 9.807 \\ \text { EBITINT } & 25.914 & 11.401 & 51.392 & 0.038 & 369.383 \\ \text { EBITDAINT } & 34.493 & 15.647 & 71.685 & 2.208 & 547.558 \\ & & & & & \text { (continued on next page) }\end{array}$


Table 3. (continued)

\begin{tabular}{lcccccc}
\hline Variable & Mean & Median & $\begin{array}{c}\text { Standard } \\
\text { deviation }\end{array}$ & Minimum & Maximum & $p$-value \\
\hline FFO & 4140.589 & 1626.167 & 6718.647 & 14.988 & 45991.667 & \\
INT & 342.962 & 118.834 & 576.459 & 2.992 & 4176.468 & \\
CASHEQ & 2697.290 & 713.283 & 4642.380 & 4.282 & 29752.667 & \\
OM & 17.992 & 17.102 & 8.935 & -0.051 & 52.755 & \\
ROC & 13.249 & 12.470 & 7.108 & -0.328 & 34.792 & \\
ROE & 21.083 & 18.777 & 13.304 & -1.688 & 96.553 & \\
ROA & 8.578 & 7.940 & 4.516 & -0.211 & 19.644 \\
RETA & 0.394 & 0.373 & 0.252 & -0.126 & 0.989 \\
CR & 1.509 & 1.337 & 0.712 & 0.518 & 4.329 & \\
QR & 1.047 & 0.933 & 0.585 & 0.262 & 3.488 & \\
CASHR & 0.453 & 0.261 & 0.503 & 0.012 & 2.455 & \\
\hline
\end{tabular}

Note: The notation ${ }^{* *}$ and $*$ indicates the significance of the F-test at the $1 \%$ and $5 \%$ levels, respectively. 
Table 4. The variable selection result. The variables were selected in sequence for OLPM by the forward selection procedure based on minimizing in-sample total error rate. The in-sample total error rate was computed using the classification scheme based on OLPM with cutoff value $1 / 2$. The newly entered variable must be able to reduce at least one percent of in-sample total error rate of the classification scheme using those already entered variables.

\begin{tabular}{|c|c|c|}
\hline Selection sequence & Variable & $\gamma_{i n}(1 / 2)$ \\
\hline 1 & RSIZE & $0.436 *$ \\
\hline 2 & SIGMA & $0.351^{*}$ \\
\hline 3 & LDC & $0.283^{*}$ \\
\hline 4 & Industry effects & $0.242 *$ \\
\hline 5 & ROE & $0.237 *$ \\
\hline 6 & $\log _{10}(\mathrm{TA})$ & 0.235 \\
\hline 7 & CR & 0.232 \\
\hline 8 & CASHR & 0.228 \\
\hline 9 & TDEBITDA & 0.228 \\
\hline 10 & $\mathrm{QR}$ & 0.228 \\
\hline 11 & EBITDAINT & 0.225 \\
\hline 12 & EXRET & 0.225 \\
\hline 13 & TDC & 0.223 \\
\hline 14 & FFO & 0.215 \\
\hline 15 & SDC & 0.215 \\
\hline 16 & KMV & 0.223 \\
\hline 17 & EM & 0.223 \\
\hline 18 & $\mathrm{OM}$ & 0.228 \\
\hline 19 & RETA & 0.232 \\
\hline 20 & INT & 0.223 \\
\hline 21 & EBITINT & 0.220 \\
\hline 22 & ROA & 0.230 \\
\hline 23 & ROC & 0.232 \\
\hline 24 & CASHEQ & 0.237 \\
\hline
\end{tabular}

Note: The notation * indicates that the variable was selected by the forward selection procedure. 
Table 5. Maximum likelihood estimates of the parameters in Models 1 and 2. Model 1 denotes OLPM with sample selection. Model 2 stands for OLPM. The variables used in each model were selected by the forward selection procedure shown in Table 4. The $p$-values refer to the Wald chi-squared tests for testing the significance of parameters. Panel A shows the results of OLPM under Models 1 and 2. Panel B gives the results of the two-class linear probit model under Model 1. Panel C presents the results for model fit test.

\begin{tabular}{|c|c|c|c|c|}
\hline \multirow{2}{*}{ Variable } & \multicolumn{2}{|c|}{ Model 1} & \multicolumn{2}{|c|}{ Model 2} \\
\hline & Coefficient & $p$-value & Coefficient & $p$-value \\
\hline \multicolumn{5}{|c|}{ Panel A: Ordered linear probit specification } \\
\hline Threshold $\delta_{2}$ & 1.918 & $0.000 * *$ & 1.923 & $0.000^{* *}$ \\
\hline Intercept $\xi$ & 7.678 & $0.000^{* *}$ & 7.639 & $0.000 * *$ \\
\hline $\mathrm{SIC}_{1}$ & 0.199 & 0.667 & 0.207 & 0.443 \\
\hline $\mathrm{SIC}_{2}$ & 0.275 & 0.466 & 0.266 & 0.167 \\
\hline $\mathrm{SIC}_{3}$ & -0.064 & 0.777 & -0.066 & 0.748 \\
\hline $\mathrm{SIC}_{4}$ & -0.683 & 0.173 & -0.691 & $0.042 *$ \\
\hline $\mathrm{SIC}_{5}$ & -0.885 & $0.016^{*}$ & -0.883 & $0.010^{* *}$ \\
\hline $\mathrm{SIC}_{6}$ & -1.208 & 0.061 & -1.197 & $0.008^{* *}$ \\
\hline RSIZE & 0.859 & 0.370 & 0.834 & $0.000^{* *}$ \\
\hline SIGMA & -37.954 & $0.000^{* *}$ & -37.750 & $0.000 * *$ \\
\hline LDC & -4.120 & $0.000^{* *}$ & -4.128 & $0.000 * *$ \\
\hline ROE & 0.015 & $0.048^{*}$ & 0.015 & $0.000^{* *}$ \\
\hline
\end{tabular}

Panel B: Two-class linear probit sample selection specification

$\begin{array}{lcc}\text { Intercept } & 2.509 & 0.000^{* *} \\ \mathrm{SIC}_{1} & -0.134 & 0.462 \\ \mathrm{SIC}_{2} & 0.178 & 0.188 \\ \mathrm{SIC}_{3} & 0.023 & 0.876 \\ \text { SIC }_{4} & 0.101 & 0.664 \\ \text { SIC }_{5} & -0.072 & 0.728 \\ \text { SIC }_{6} & -0.270 & 0.342 \\ \text { RSIZE } & 0.505 & 0.000^{* *} \\ \text { SIGMA } & -5.636 & 0.000^{* *} \\ \text { LDC } & -0.069 & 0.734 \\ \text { ROE } & -0.001 & 0.174\end{array}$

Panel C: Model fit test

Chi-squared

$\begin{array}{lcccc}\text { statistic } & 0.012 & 0.914 & 399.014 & 0.000^{* *} \\ \text { d.f. } & 1 & & 10 & \end{array}$

$\begin{array}{lll}\text { corr } & 0.095 & 0.981\end{array}$

Note: The notation ${ }^{* *}$ and $*$ indicates the significance of the test at the $1 \%$ and $5 \%$ levels, respectively. The notation d.f. and corr stands for the degree of freedom and the correlation coefficient between the error term in OLPM and that in the two-class linear probit model, respectively. 
Table 6. Classification results obtained from the 413 estimation companies. Panels A and B show the results obtained by the two prediction rules based on OLPM and OSPM in the case of $u=1$, respectively.

\begin{tabular}{|c|c|c|c|}
\hline \multirow{2}{*}{ True category } & \multicolumn{3}{|c|}{ Classified category } \\
\hline & $\{$ Below BBB $\}$ & $\{\mathrm{BBB}\}$ & $\{\mathrm{AAA}, \mathrm{AA}, \mathrm{A}\}$ \\
\hline \multicolumn{4}{|c|}{ Panel A: OLPM with $u=1$} \\
\hline$\{$ Below BBB $\}$ & 123 & 23 & 1 \\
\hline$\{\mathrm{BBB}\}$ & 16 & 122 & 18 \\
\hline$\{\mathrm{AAA}, \mathrm{AA}, \mathrm{A}\}$ & 4 & 35 & 71 \\
\hline \multicolumn{4}{|c|}{$\alpha_{\text {in }}=(23+1+18) / 413=0.102, \quad \beta_{\text {in }}=(16+4+35) / 413=0.133, \quad \gamma_{\text {in }}=0.102+0.133=0.235$} \\
\hline \multicolumn{4}{|c|}{ Panel B: OSPM with $u=1$} \\
\hline$\{$ Below BBB $\}$ & 126 & 21 & 0 \\
\hline$\{\mathrm{BBB}\}$ & 17 & 129 & 10 \\
\hline$\{\mathrm{AAA}, \mathrm{AA}, \mathrm{A}\}$ & 4 & 43 & 63 \\
\hline \multicolumn{4}{|c|}{$\alpha_{\text {in }}=(21+0+10) / 413=0.075, \quad \beta_{\text {in }}=(17+4+43) / 413=0.155, \quad \gamma_{\text {in }}=0.075+0.155=0.230$} \\
\hline
\end{tabular}


Table 7. Prediction results obtained from the 366 holdout companies. Panels A and B show the results obtained by the two prediction rules based on OLPM and OSPM in the case of $u=1$, respectively.

\begin{tabular}{|c|c|c|c|}
\hline \multirow{2}{*}{ True category } & \multicolumn{3}{|c|}{ Predicted category } \\
\hline & $\{$ Below BBB $\}$ & $\{\mathrm{BBB}\}$ & $\{\mathrm{AAA}, \mathrm{AA}, \mathrm{A}\}$ \\
\hline \multicolumn{4}{|c|}{ Panel A: OLPM with $u=1$} \\
\hline$\{$ Below BBB $\}$ & 207 & 35 & 9 \\
\hline$\{\mathrm{BBB}\}$ & 16 & 54 & 8 \\
\hline$\{\mathrm{AAA}, \mathrm{AA}, \mathrm{A}\}$ & 2 & 18 & 17 \\
\hline \multicolumn{4}{|c|}{$\alpha_{\text {out }}=(35+9+8) / 366=0.142, \quad \beta_{\text {out }}=(16+2+18) / 366=0.098$} \\
\hline \multicolumn{4}{|c|}{ Panel B: OSPM with $u=1$} \\
\hline$\{$ Below BBB $\}$ & 223 & 26 & 2 \\
\hline$\{\mathrm{BBB}\}$ & 15 & 60 & 3 \\
\hline$\{\mathrm{AAA}, \mathrm{AA}, \mathrm{A}\}$ & 4 & 19 & 14 \\
\hline \multicolumn{4}{|c|}{$\alpha_{\text {out }}=(26+2+3) / 366=0.085, \quad \beta_{\text {out }}=(15+4+19) / 366=0.104, \quad \gamma_{\text {out }}=0.085+0.104=0.189$} \\
\hline
\end{tabular}

